\title{
Analytical Green's functions for continuum spectra
}

\author{
Eugenio Megías ${ }^{a}$ and Mariano Quirós ${ }^{b}$ \\ ${ }^{a}$ Departamento de Física Atómica, Molecular y Nuclear \\ and Instituto Carlos I de Física Teórica y Computacional, Universidad de Granada, \\ Avenida de Fuente Nueva s/n, 18071 Granada, Spain \\ ${ }^{b}$ Institut de Física d'Altes Energies (IFAE) \\ and The Barcelona Institute of Science and Technology (BIST), \\ Campus UAB, 08193 Bellaterra, Barcelona, Spain \\ E-mail: emegias@ugr.es, quiros@ifae.es
}

ABSTRACT: Green's functions with continuum spectra are a way of avoiding the strong bounds on new physics from the absence of new narrow resonances in experimental data. We model such a situation with a five-dimensional model with two branes along the extra dimension $z$, the ultraviolet (UV) and the infrared (IR) one, such that the metric between the UV and the IR brane is $\mathrm{AdS}_{5}$, thus solving the hierarchy problem, and beyond the IR brane the metric is that of a linear dilaton model, which extends to $z \rightarrow \infty$. This simplified metric, which can be considered as an approximation of a more complicated (and smooth) one, leads to analytical Green's functions (with a mass gap $m_{g} \sim \mathrm{TeV}$ and a continuum for $s>m_{g}^{2}$ ) which could then be easily incorporated in the experimental codes. The theory contains Standard Model gauge bosons in the bulk with Neumann boundary conditions in the UV brane. To cope with electroweak observables the theory is also endowed with an extra custodial gauge symmetry in the bulk, with gauge bosons with Dirichlet boundary conditions in the UV brane, and without zero (massless) modes. All Green's functions have analytical expressions and exhibit poles in the second Riemann sheet of the complex plane at $s=M_{n}^{2}-i M_{n} \Gamma_{n}$, denoting a discrete (infinite) set of broad resonances with masses $\left(M_{n}\right)$ and widths $\left(\Gamma_{n}\right)$. For gauge bosons with Neumann or Dirichlet boundary conditions, the masses and widths of resonances satisfy the (approximate) equation $s=$ $-4 m_{g}^{2} \mathcal{W}_{n}^{2}[ \pm(1+i) / 4]$, where $\mathcal{W}_{n}$ is the $n$-th branch of the Lambert function.

Keywords: Phenomenology of Field Theories in Higher Dimensions, Phenomenology of Large extra dimensions

ArXiv EPrint: 2106.09598 


\section{Contents}

1 Introduction 1

2 The five-dimensional model $\quad 3$

2.1 The gravitational background 4

$\begin{array}{lll}2.2 & \text { The gauge sector } & 7\end{array}$

3 Standard model massless gauge bosons $\quad 8$

3.1 General Green's functions 9

3.2 Brane-to-brane Green's functions 11

$\begin{array}{ll}3.3 & \text { Green's functions in the complex plane and resonances } \\ \end{array}$

$\begin{array}{ll}3.4 \text { Spectral functions } & 19\end{array}$

3.5 Unparticle vs. resonant contribution to Green's functions 22

4 Gauge bosons with Dirichlet boundary condition 23

5 Electroweak precision observables $\quad 26$

6 Conclusions and outlook $\quad 29$

$\begin{array}{ll}\text { A Standard model massive gauge bosons } & 30\end{array}$

\section{Introduction}

The Standard Model (SM) of electroweak (EW) and strong interactions has been put on solid grounds by past and current experimental data, collected at e.g. the Large Electron Positron (LEP) or the Large Hadron Collider (LHC) [1, 2]. In spite of the lack of clear deviations in particle physics experiments, there is a number of observational facts (dark matter, baryon asymmetry of the universe, ... ), and theoretical drawbacks (hierarchy problem, ... ) which cannot be coped by the SM and demand some ultraviolet (UV) completion of the theory. This has motivated a plethora of beyond the SM (BSM) models.

One of the most successful BSM models is the Randall-Sundrum (RS) model, proposed in 1999 [3], where the hierarchy between the four-dimensional (4D) Planck scale $M_{\mathrm{Pl}}$ and the $\mathrm{TeV}$ scale is solved by means of a warped fifth dimension and two branes, the UV brane and the infrared (IR) brane. Associated with each SM field, the theory predicts a discrete spectrum made out of towers of composite discrete states known as KaluzaKlein (KK) states, with masses in the $\mathrm{TeV}$ range. The elusiveness of isolated and narrow resonances in direct searches at the LHC $[4,5]$, led people to explore different solutions to the hierarchy problem that could escape present detection, as e.g. the presence of broad resonances [6]. Some other exploring scenarios include the clockwork models, as well as their $5 \mathrm{D}$ continuum limit $[7,8]$, the linear dilaton models (LDM) $[9,10]$ and the Little String 
theories [11], which predict discrete spectra with a $\mathrm{TeV}$ mass gap and a mass separation between modes $\sim 30 \mathrm{GeV}$.

A new scenario has been recently proposed, in which there appears a $\mathrm{TeV}$ mass gap followed by a continuum of resonances heavier than the mass gap [12-16]. These models are characterized by the absence of the IR boundary (replaced by an admissible singularity of the metric), ${ }^{1}$ and the gapped continuum spectrum is present when the behavior of the bulk potential of the stabilizing (canonically normalized) 5D scalar field $\phi$ is given, in the limit $\phi \rightarrow \infty$, by the critical behavior, $V(\phi) \propto \exp \left(\sqrt{\frac{2}{3 M_{5}^{3}}} \phi\right)$, where $M_{5}$ is the $5 \mathrm{D}$ Planck scale [22]. The behavior of the metric near the UV boundary is $\mathrm{AdS}_{5}$, thus giving a connection with the RS model in this regime, and allowing for a holographic interpretation of the model and relating it with unparticles [23, 24]. The model includes an IR brane, where the Higgs (a mesonic doublet) is localized and which triggers EW symmetry breaking, while the fifth dimension extends beyond the IR brane till the singularity.

In a recent publication we have presented the results of the Green's functions by focusing on the holographic method, which is convenient for the computation of UV-to-UV brane propagators [13]. In this paper we will use a different approach based on the direct computation of the Green's functions from the inhomogeneous equations of motion with appropriate boundary conditions. We will use for that a simplified metric which behaves like $\mathrm{AdS}_{5}$ between the UV and the IR branes, and like the metric of the LDM, between the IR brane and the singularity. This will allow us to compute the Green's functions of fields propagating at any point in the bulk. In addition, the model presented in this work is simple enough to lead to analytical formulas while, at the same time, sharing all the desirable features of a model which leads to a gapped continuum spectrum as discussed in ref. [13].

This theory can be considered as a modelization of $4 \mathrm{D}$ theories with continuum spectra and a mass gap, as can be the case of unparticle theories and Unhiggs theories, which share similar features and whose phenomenology has been extensively studied in a number of papers [25-32]. In order to protect EW precision observables we will need to introduce an extra custodial gauge symmetry. Although more realistic models can be introduced, we will just consider the simplest model [33] where the gauge symmetry for the EW sector in the bulk is $\mathrm{SU}(2)_{L} \times \mathrm{SU}(2)_{R} \times \mathrm{U}(1)_{B-L}$ which breaks to $\mathrm{U}(1)_{Y}$ by the UV boundary conditions, while it remains unbroken in the IR brane.

The outline of this paper is as follows. We introduce in section 2 the general formalism for the 5D action, including the gravitational background and the gauge sector which will be used throughout the rest of the paper. The Green's functions and the spectral functions for the massless gauge bosons are studied in section 3. In particular the Green's functions in the complex $s$ plane are studied, which lead to complex poles in the second Riemann sheet, interpreted as broad resonances. The similar analysis for the SM massive gauge bosons $W, Z$, including the Green's functions, spectral functions and resonances, is postponed to appendix A. The computation of the Green's functions for gauge bosons with Dirichlet boundary condition in the UV brane is addressed in section 4 . Finally we

\footnotetext{
${ }^{1}$ Identifying the IR brane with the manifold boundary gives rise to a different class of models with a different phenomenology, which has been explored in a number of papers, refs. [17-21].
} 
present in section 5 the prediction of the model for the electroweak precision observables. We conclude with a discussion of our results, and an outlook toward future directions in section 6 .

\section{The five-dimensional model}

We consider a slice of $5 \mathrm{D}$ space-time between a brane at the value $y=y_{0}=0$ in proper coordinates, the UV brane, and an admissible singularity placed at $y=y_{s}$, a value which is determined dynamically. In addition, we will introduce an IR brane, at $y=y_{1}<y_{s}$, responsible for electroweak breaking, where we will assume the Higgs sector is localized.

The $5 \mathrm{D}$ action of the model, including the stabilizing bulk scalar $\phi(x, y)$, with mass dimension $3 / 2$, reads as

$$
\begin{aligned}
S= & \int d^{5} x \sqrt{\left|\operatorname{det} g_{M N}\right|}\left[-\frac{1}{2 \kappa^{2}} R+\frac{1}{2} g^{M N}\left(\partial_{M} \phi\right)\left(\partial_{N} \phi\right)-V(\phi)\right] \\
& -\sum_{\alpha} \int_{B_{\alpha}} d^{4} x \sqrt{\left|\operatorname{det} \bar{g}_{\mu \nu}\right|} \lambda_{\alpha}(\phi)-\frac{1}{\kappa^{2}} \int_{B_{0}} d^{4} x \sqrt{\left|\operatorname{det} \bar{g}_{\mu \nu}\right|} K_{0},
\end{aligned}
$$

where $\kappa^{2}=1 /\left(2 M_{5}^{3}\right)$, with $M_{5}$ being the 5D Planck scale, $V(\phi)$ and $\lambda_{\alpha}(\phi)$ are the bulk and brane potentials of the scalar field $\phi$, and the index $\alpha=0(\alpha=1)$ refers to the UV (IR) brane. We will assume a $\mathbb{Z}_{2}$ symmetry $(y \rightarrow-y)$ across the UV brane, which translates into boundary conditions on the fields, while we will impose matching conditions for bulk fields across the IR brane. Note that the fifth dimension continues beyond the IR brane until the singularity. The IR brane is responsible for the generation of the $\sim \mathrm{TeV}$ scale, and contains the brane Higgs potential which spontaneously breaks the electroweak symmetry, thus solving the hierarchy problem, as we will see.

The parameter $\kappa^{2}$, can be traded by the parameter $N$ in the dual theory by the relation [34] $N^{2} \simeq \frac{8 \pi^{2} \ell^{3}}{\kappa^{2}}$, where $\ell \equiv 1 / k$ is a parameter of the order of the Planck length, which determines the value of the $5 \mathrm{D}$ curvature. The metric $g_{M N}$ is defined in proper coordinates by

$$
d s^{2}=g_{M N} d x^{M} d x^{N} \equiv e^{-2 A(y)} \eta_{\mu \nu} d x^{\mu} d x^{\nu}-d y^{2},
$$

so that in eq. (2.1) the $4 \mathrm{D}$ induced metric is $\bar{g}_{\mu \nu}=e^{-2 A(y)} \eta_{\mu \nu}$, where the Minkowski metric is given by $\eta_{\mu \nu}=\operatorname{diag}(1,-1,-1,-1)$. The last term in eq. (2.1) is the usual GibbonsHawking-York boundary term $[35,36]$, where $K_{0}$ is the extrinsic UV curvature. In terms of the metric of eq. (2.2) the extrinsic curvature term reads as [37] $K_{0}=-4 A^{\prime}\left(y_{0}\right)$.

The equations of motion (EoM) read then as $^{2}$

$$
\begin{aligned}
A^{\prime \prime} & =\frac{\kappa^{2}}{3} \phi^{\prime 2}+\frac{\kappa^{2}}{3} \sum_{\alpha} \lambda_{\alpha}(\phi) \delta\left(y-y_{\alpha}\right), \\
A^{\prime 2} & =-\frac{\kappa^{2}}{6} V(\phi)+\frac{\kappa^{2}}{12} \phi^{\prime 2}, \\
\phi^{\prime \prime}-4 A^{\prime} \phi^{\prime} & =V^{\prime}(\phi)+\sum_{\alpha} \lambda_{\alpha}^{\prime}(\phi) \delta\left(y-y_{\alpha}\right) .
\end{aligned}
$$

\footnotetext{
${ }^{2}$ From here on the prime symbol $\left({ }^{\prime}\right)$ will stand for the derivative of a function with respect to its argument, and the dot symbol $\left({ }^{*}\right)$ derivative only with respect to the conformal coordinate $z$ related to $y$ by $d y=e^{-A} d z$.
} 
The EoM in the bulk can also be written in terms of the superpotential $W(\phi)$ as [38]

$$
\phi^{\prime}(y)=\frac{1}{2} \frac{\partial W}{\partial \phi}, \quad A^{\prime}(y)=\frac{\kappa^{2}}{6} W
$$

and

$$
V(\phi)=\frac{1}{8}\left(\frac{\partial W}{\partial \phi}\right)^{2}-\frac{\kappa^{2}}{6} W^{2}(\phi) .
$$

Due to the $\mathbb{Z}_{2}$ symmetry across the UV brane, the localized terms impose the following boundary conditions in the UV

$$
W\left(\phi\left(y_{0}\right)\right)=\lambda_{0}\left(\phi\left(y_{0}\right)\right), \quad W^{\prime}\left(\phi\left(y_{0}\right)\right)=\lambda_{0}^{\prime}\left(\phi\left(y_{0}\right)\right) .
$$

In addition, the IR brane leads to the following jumping conditions

$$
\Delta W\left(\phi\left(y_{1}\right)\right)=2 \lambda_{1}\left(\phi\left(y_{1}\right)\right), \quad \Delta W^{\prime}\left(\phi\left(y_{1}\right)\right)=2 \lambda_{1}^{\prime}\left(\phi\left(y_{1}\right)\right),
$$

where $\Delta X$ is the jump when crossing the brane.

In the following we will impose continuity conditions for $W(\phi)$ and $W^{\prime}(\phi)$. Simple brane potentials satisfying the boundary conditions of eq. (2.8), the jumping conditions of eq. (2.9) with $\Delta W\left(\phi\left(y_{1}\right)\right)=\Delta W^{\prime}\left(\phi\left(y_{1}\right)\right)=0$, and fixing dynamically the values $v_{\alpha}$ of $\phi$ at the branes, i.e. $v_{\alpha}=\phi\left(y_{\alpha}\right)$, are given by

$$
\lambda_{0}(\phi)=W(\phi)+\frac{1}{2} \gamma_{0}\left(\phi-v_{0}\right)^{2}, \quad \lambda_{1}(\phi)=\frac{1}{2} \gamma_{1}\left(\phi-v_{1}\right)^{2} .
$$

This formalism has been extensively discussed in e.g. refs. [39, 40].

\subsection{The gravitational background}

We will provide in this section a particular realization of the gravitational background that will be used in the present work. As we will see, the model is simple enough to obtain analytical results for the Green's function in the forthcoming sections, but it contains all the ingredients needed to study the physics of the gapped continuum spectra, while solving the hierarchy problem à la RS.

A simple model solving the hierarchy problem and with a continuum spectrum was already characterized in ref. [13] by the superpotential

$$
W(\phi)=\frac{6 k}{\kappa^{2}}\left(1+e^{\kappa \phi / \sqrt{3}}\right)
$$

or the corresponding bulk potential $V(\phi)$

$$
V(\phi)=-\frac{6 k^{2}}{\kappa^{2}}\left[1+2 e^{\kappa \phi / \sqrt{3}}+\frac{3}{4} e^{2 \kappa \phi / \sqrt{3}}\right] .
$$

After solving the EoM, the background value of the scalar field $\phi$ and warp factor $A(y)$ are given by

$$
\phi(y)=-\frac{\sqrt{3}}{\kappa} \log \left[k\left(y_{s}-y\right)\right], \quad A(y)=k y-\log \left(\frac{y_{s}-y}{y_{s}}\right),
$$


where $y_{s}$ is the location of the singularity in proper coordinates, such that after fixing the value of the field $\phi$ in the branes, at $y=y_{\alpha}$, by brane potentials $\lambda_{\alpha}(\phi)$, dynamically fixing $\phi\left(y_{\alpha}\right)=v_{\alpha}$, the brane and singularity distances are fixed by

$$
k y_{s}=e^{-\kappa v_{0} / \sqrt{3}}, \quad k y_{1}=e^{-\kappa v_{0} / \sqrt{3}}-e^{-\kappa v_{1} / \sqrt{3}} .
$$

As we have seen in ref. [13], the value of the gap and the warped $k$ scale at the IR brane

$$
\rho \equiv e^{-A\left(y_{1}\right)} k,
$$

of the $\mathrm{TeV}$ size, as required to solve the hierarchy problem, should be of the same order of magnitude. The further requirement of identification of both scales leads to the extra condition

$$
k\left(y_{s}-y_{1}\right)=1
$$

which amounts to the choice $v_{1}=0$, which can be taken without loss of generality. In the rest of this paper the relation (2.16) will be adopted.

In particular the warp factor $A(y)$ behaves like the RS-metric between the UV and IR branes $\left(0<k y \lesssim k y_{1}\right), A(y) \simeq k y$, while it behaves like $A(y) \simeq-\log \left(\frac{y_{s}-y}{y_{s}}\right)$ between the IR brane and the singularity $\left(y \gtrsim y_{1}\right)$. We can then approximate the exact metric by the approximate one

$$
A(y) \simeq k y \Theta\left(y_{1}-y\right)+\left[k y_{1}-\log \left(k y_{s}-k y\right)\right] \Theta\left(y-y_{1}\right),
$$

where the step function is $\Theta(x)=1$ ( 0$)$ for $x>0(x \leq 0)$. Comparison between both, the exact (2.13) and approximate (2.17), metrics is done in the plot of figure 1. As we can see the approximate solution of eq. (2.17) behaves like the exact one in the relevant regions of the parameter space and moreover, as we will see in the next sections, it will allow for analytical solutions to the Green's functions which, otherwise, could only be computed numerically [13].

The approximate metric of eq. (2.17) can arise from the (approximate) superpotential

$$
W(\phi) \simeq \frac{6 k}{\kappa^{2}} \Theta\left(v_{1}-\phi\right)+\frac{6 k}{\kappa^{2}} e^{\kappa\left(\phi-v_{0}\right) / \sqrt{3}} \Theta\left(\phi-v_{1}\right),
$$

which leads to the following profile for the field $\phi$

$$
\phi(y) \simeq v_{0} \Theta\left(y_{1}-y\right)+\left[v_{0}-\frac{\sqrt{3}}{\kappa} \log \left(k y_{s}-k y\right)\right] \Theta\left(y-y_{1}\right) .
$$

It can be useful to define the metric in conformally flat coordinates defined by $d s^{2}=$ $e^{-2 A(z)}\left(\eta_{\mu \nu} d x^{\mu} d x^{\nu}-d z^{2}\right)$. Comparison with eq. (2.2) leads to the relation between conformal and proper coordinates, $z^{\prime}(y)=e^{A(y)}$, that can be solved to obtain

$$
z(y) \simeq \frac{e^{k y}}{k} \Theta\left(y_{1}-y\right)+\frac{e^{k y_{1}}}{k}\left[1-\log \left(k y_{s}-k y\right)\right] \Theta\left(y-y_{1}\right),
$$




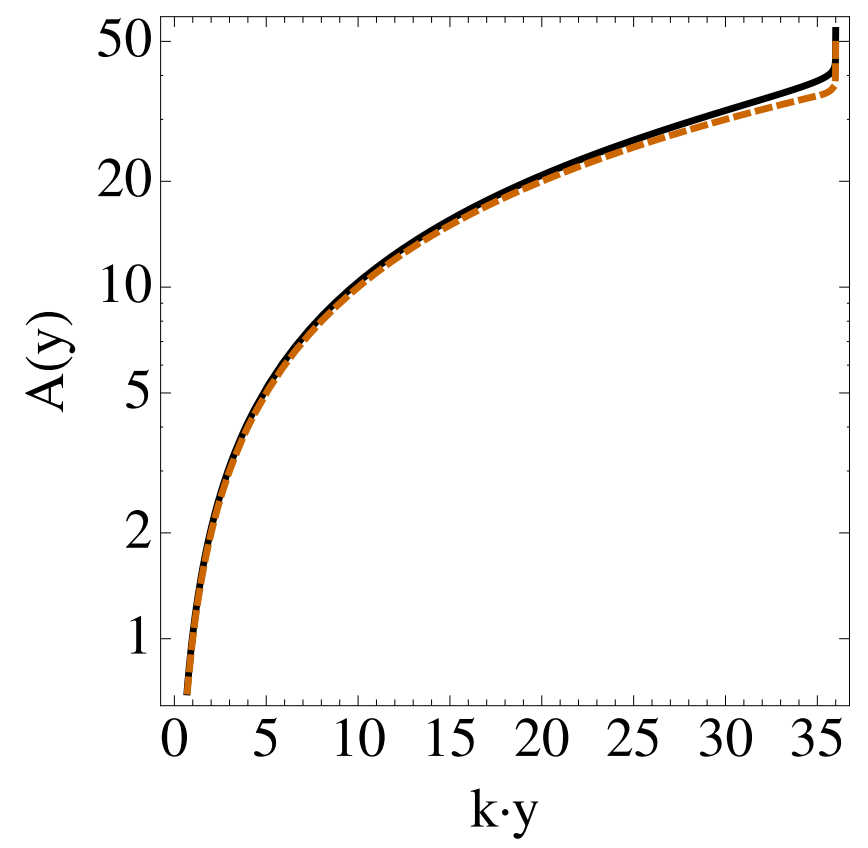

Figure 1. The warp factor $A(y)$ for the exact solution (solid line) of eq. (2.13) and the approximate solution (dashed line) of eq. (2.17). We have considered $k y_{s}=36$.

so that $z_{s}=\lim _{y \rightarrow y_{s}} z(y)=\infty$. Similarly one finds the following expression for the scalar field as a function of the conformal coordinate

$$
\phi(z) \simeq v_{0} \Theta\left(z_{1}-z\right)+\left[v_{0}+\frac{\sqrt{3}}{\kappa} \rho\left(z-z_{1}\right)\right] \Theta\left(z-z_{1}\right),
$$

where we have defined the quantities

$$
z_{0} \equiv 1 / k \quad \text { and } \quad z_{1} \equiv 1 / \rho .
$$

Note that we have fixed the integration constant by considering in the UV brane $z(y=$ $0)=1 / k$. Finally, one can write the warp factor in terms of the conformal coordinate, leading to

$$
A(z) \simeq \log (k z) \Theta\left(z_{1}-z\right)+\left[\log \left(k z_{1}\right)+\rho\left(z-z_{1}\right)\right] \Theta\left(z-z_{1}\right) .
$$

In this paper we will indistinctly use proper $(y)$ or conformal $(z)$ coordinates.

The value of $M_{5}$ is determined by the relation of $M_{5}$ and $k$ with the $4 \mathrm{D}$ rationalized Planck mass, $M_{\mathrm{Pl}}=2.4 \times 10^{18} \mathrm{GeV}$, via the expression

$$
\kappa^{2} M_{\mathrm{Pl}}^{2}=\int_{0}^{y_{s}} d y e^{-2 A(y)} \quad \Longrightarrow \quad M_{5}^{3} \simeq k M_{\mathrm{Pl}}^{2} .
$$

Taking into account that $k \lesssim M_{5}$, this yields $M_{5} \simeq \mathcal{O}\left(M_{\mathrm{Pl}}\right)$ so that the Planck scale turns out to be the fundamental scale of the theory, and the $\mathrm{TeV}$ is a derived scale after warping. This situation contrasts with the discrete $\mathrm{LDM}$, in which $M_{5} \simeq \rho \simeq \mathrm{TeV}$ are fundamental scales $[9,10]$ (see the discussion in ref. [15]). 


\subsection{The gauge sector}

As it will become clear in this paper, as the theory is RS between the UV and IR branes, in order to protect electroweak observables from going out of control, the SM gauge group has to be extended with an extra custodial gauge symmetry $\mathrm{SU}(2)_{R}$ [33]. The custodial model is then based on the bulk gauge group [33, 41-44]

$$
\mathrm{SU}(3)_{c} \otimes \mathrm{SU}(2)_{L} \otimes \mathrm{SU}(2)_{R} \otimes \mathrm{U}(1)_{X}
$$

where $X \equiv B-L$, with 5 D gauge bosons $\left(G, W_{L}, W_{R}, X\right)$, with mass dimension $3 / 2$, and $5 \mathrm{D}$ couplings $\left(g_{c}, g_{L}, g_{R}, g_{X}\right)$, with mass dimension $-1 / 2$.

The breaking $\mathrm{SU}(2)_{R} \otimes \mathrm{U}(1)_{X} \rightarrow \mathrm{U}(1)_{Y}$, where $Y$ is the SM hypercharge, with gauge boson $B$ and coupling $g_{Y}$, is done in the UV brane by boundary conditions. Therefore the gauge fields $\left(W_{L}^{a}, W_{R}^{a}, X\right)$ define $\left(W_{L}^{a}, W_{R}^{1,2}, B, Z_{R}\right)$, with (UV, IR) boundary conditions, as

$$
\begin{array}{cc}
W_{L}^{a}(a=1,2,3), & (+,+) \\
B=\frac{g_{X} W_{R}^{3}+g_{R} X}{\sqrt{g_{R}^{2}+g_{X}^{2}}}, & (+,+) \\
W_{R}^{1,2}, & (-,+) \\
Z_{R}=\frac{g_{R} W_{R}^{3}-g_{X} X}{\sqrt{g_{R}^{2}+g_{X}^{2}}} . & (-,+)
\end{array}
$$

The $\mathrm{SU}(2)_{L} \otimes \mathrm{SU}(2)_{R}$ symmetry is unbroken in the IR brane, where all composite states are localized, such that the custodial symmetry is exact.

The covariant derivative for fermions is

$$
\not D=\not \partial-i\left[g_{L} \sum_{a=1}^{3} W_{L}^{a} T_{L}^{a}+g_{R} \sum_{b=1}^{2} W_{R}^{b} T_{R}^{b}+g_{Y} \not B Y+g_{Z_{R}} Z_{R} Q_{Z_{R}}\right],
$$

where $g_{Y}$ and $g_{Z_{R}}$ are defined in terms of $g_{R}$ and $g_{X}$ as

$$
g_{Y} \equiv \frac{g_{R} g_{X}}{\sqrt{g_{R}^{2}+g_{X}^{2}}}, \quad g_{Z_{R}} \equiv \sqrt{g_{R}^{2}+g_{X}^{2}},
$$

and the hypercharge $Y$ and the charge $Q_{Z_{R}}$ are defined by

$$
Y \equiv T_{R}^{3}+Q_{X}, \quad Q_{Z_{R}} \equiv \frac{g_{R}^{2} T_{R}^{3}-g_{X}^{2} Q_{X}}{g_{R}^{2}+g_{X}^{2}},
$$

with $Q_{X} \equiv(B-L) / 2$.

Electroweak symmetry breaking is triggered in the IR brane by the bulk Higgs bidoublet

$$
\mathcal{H}=\left(\begin{array}{cc}
H_{2}^{0} & H_{1}^{+} \\
H_{2}^{-} & H_{1}^{0}
\end{array}\right), \quad Q_{X}=0,
$$

where the rows transform under $\mathrm{SU}(2)_{L}$ and the columns under $\mathrm{SU}(2)_{R}$. We will denote their VEVs as $\left\langle H_{2}^{0}\right\rangle \equiv v_{2} / \sqrt{2}$ and $\left\langle H_{1}^{0}\right\rangle \equiv v_{1} / \sqrt{2}$, so that we will introduce the angle $\beta$ as, $\cos \beta=v_{1} / v$ and $\sin \beta=v_{2} / v$, with $v \equiv \sqrt{v_{1}^{2}+v_{2}^{2}}$. 
One can rotate to the gauge boson mass eigenstates by considering the angle $\theta_{L} \equiv \theta_{W}$, which is the usual weak mixing angle, and $\theta_{R}$, defined as

$$
\cos \theta_{R} \equiv \frac{g_{R}}{\sqrt{g_{R}^{2}+g_{X}^{2}}}, \quad \sin \theta_{R} \equiv \frac{g_{X}}{\sqrt{g_{R}^{2}+g_{X}^{2}}} .
$$

Using eq. (2.31) and (2.34) one finds $\sin \theta_{R}=g_{Y} / g_{R}<1$.

As for fermions, left-handed ones are in $\mathrm{SU}(2)_{L}$ bulk doublets as in the SM

$$
Q_{L}^{i}=\left(\begin{array}{c}
u_{L} \\
d_{L}
\end{array}\right)^{i}, \quad L_{L}^{i}=\left(\begin{array}{c}
\nu_{L} \\
e_{L}
\end{array}\right)^{i},
$$

where the index $i$ runs over the three generations. On the other hand, as $\mathrm{SU}(2)_{R}$ is a symmetry of the bulk, right-handed fermions $F_{R}^{i}(F=e, u, d)$ should appear in doublets of $\mathrm{SU}(2)_{R}, F_{R}^{i}=\left(f_{R}, f_{R}^{\prime}\right)^{i}$. However, as $\mathrm{SU}(2)_{R}$ is broken by the orbifold conditions on the UV brane it means, for bulk right-handed fermions, that one component of the doublet must be even, under the orbifold $\mathbb{Z}_{2}$ parity, and has a zero mode, while the other component of the doublet must be odd, and thus without any zero mode. We thus need to double the SM right-handed fermions in the bulk.

\section{Standard model massless gauge bosons}

In this section we will compute Green's functions for massless SM gauge bosons $A_{\mu}$ (i.e. the SM photon and gluon). The Lagrangian for massless gauge bosons is ${ }^{3}$

$$
\mathcal{L}=\int_{0}^{y_{s}} d y\left[-\frac{1}{4} \operatorname{tr} F_{\mu \nu} F^{\mu \nu}-\frac{1}{2} e^{-2 A} \operatorname{tr} A_{\mu}^{\prime} A_{\mu}^{\prime}\right],
$$

where the trace is over gauge indices. After Fourier transforming the coordinates $x^{\mu}$ into momenta $p^{\mu}$ we can make the field decomposition $A_{\mu}(p, y)=f_{A}(y) A_{\mu}(p) / \sqrt{y_{s}}$, and the EoM of the fluctuations is given by [17]

$$
p^{2} f_{A}(y)+\partial_{y}\left(e^{-2 A} \partial_{y} f_{A}(y)\right)=0 .
$$

In conformal coordinates, and after rescaling the field by $f_{A}(z)=e^{A(z) / 2} \hat{f}_{A}(z)$, we obtain the Schrödinger like form for the EoM

$$
-\ddot{\hat{f}}_{A}(z)+V_{A}(z) \hat{f}_{A}(z)=p^{2} \hat{f}_{A}(z)
$$

where the effective Schrödinger potential is

$$
V_{A}(z)=\frac{1}{4} \dot{A}^{2}(z)-\frac{1}{2} \ddot{A}(z) .
$$

Plugging eq. (2.23) into this equation, we find the following result for the effective potential

$$
V_{A}(z)=\left\{\begin{array}{cc}
3 /\left(4 z^{2}\right) & z \leq z_{1} \\
\rho^{2} / 4 & z_{1}<z
\end{array}\right.
$$

\footnotetext{
${ }^{3}$ We are using in this section the gauge $A_{5}=0$.
} 
where $z_{1} \equiv 1 / \rho$ and $\rho$ is defined in eq. $(2.15) .{ }^{4}$ We can see that in the IR regime the potential is constant

$$
V_{A}(z) \underset{z>z_{1}}{=} m_{g}^{2}, \quad \text { with } \quad m_{g}=\frac{\rho}{2} .
$$

We thus find the existence of a mass gap of the potential, which will translate into a gap followed by a continuum KK spectrum.

\subsection{General Green's functions}

We will now compute the Green's functions for gauge bosons propagating in the bulk of the 5D space-time from $y$ to $y^{\prime}$, where both $y$ and $y^{\prime}$ are considered arbitrary. To compute the Green's function we have to solve an inhomogeneous version of the EoM eq. (3.2). This is given by

$$
p^{2} G_{A}\left(y, y^{\prime} ; p\right)+\partial_{y}\left(e^{-2 A} \partial_{y} G_{A}\left(y, y^{\prime} ; p\right)\right)=\delta\left(y-y^{\prime}\right),
$$

where the derivatives are with respect to the variable $y$. After fixing the value of $y^{\prime}$, we can divide the $y$ space into the following domains: $i$ ) Region I: $0 \leq y \leq y^{\prime}$, ii) Region II: $y^{\prime}<y \leq y_{1}$, and, iii) Region III: $y_{1}<y<y_{s}$; where we are assuming $y^{\prime}<y_{1} .{ }^{5}$ When doing so, we find the general solution

$$
G_{A}\left(y, y^{\prime} ; p\right)=\left\{\begin{array}{cl}
C_{1}^{I} \cdot e^{k y} J_{1}\left(\frac{e^{k y}}{k} p\right)+C_{2}^{I} \cdot e^{k y} Y_{1}\left(\frac{e^{k y}}{k} p\right) & \text { Region I } \\
C_{1}^{I I} \cdot e^{k y} J_{1}\left(\frac{e^{k}}{k} p\right)+C_{2}^{I I} \cdot e^{k y} Y_{1}\left(\frac{e^{k y}}{k} p\right) & \text { Region II } \\
C_{1}^{I I I} \cdot\left(y_{s}-y\right)^{\frac{1}{2} \Delta_{A}^{-}}+C_{2}^{I I I} \cdot\left(y_{s}-y\right)^{\frac{1}{2} \Delta_{A}^{+}} & \text {Region III }
\end{array}\right.
$$

where $J_{1}(x)$ and $Y_{1}(x)$ are Bessel functions of the first and second kind, respectively, and

$$
\Delta_{A}^{ \pm} \equiv \pm \delta_{A}-1, \quad \delta_{A}=\sqrt{1-4 p^{2} / \rho^{2}} .
$$

Unless otherwise stated, the square root will be considered in the first Riemann sheet. ${ }^{6}$ For time-like momenta, $p^{2}>0$, we will adopt the usual prescription $p^{2} \rightarrow p^{2}+i \epsilon$, so that for real values of $p$ above the mass gap, $p>m_{g}, \delta_{A}=-i \sqrt{4 p^{2} / \rho^{2}-1}$. For space-like momenta $p^{2}<0, p \equiv i|p|$, then $\delta_{A}=\sqrt{1+4|p|^{2} / \rho^{2}}$ which is always positive.

The solution of eq. (3.8) involves six arbitrary constants $C_{i}^{I, I I, I I I}(i=1,2)$, i.e. two constants per region. The Green's functions are subject to the following boundary and matching conditions

$$
\begin{aligned}
\left(\partial_{y} G_{A}\right)\left(y_{0}\right) & =0, & \Delta G_{A}\left(y^{\prime}\right) & =0, & \Delta\left(\partial_{y} G_{A}\right)\left(y^{\prime}\right)=e^{2 A\left(y^{\prime}\right)}, \\
\Delta G_{A}\left(y_{1}\right) & =0, & \Delta\left(\partial_{y} G_{A}\right)\left(y_{1}\right) & =0, &
\end{aligned}
$$

\footnotetext{
${ }^{4}$ Note that $V_{A}(z)$ is discontinuous at $z=z_{1}$, and given eq. (3.3) this induces a discontinuity in $\ddot{\hat{f}}_{A}\left(z_{1}\right)$. Nevertheless $\hat{f}_{A}(z)$ and $\dot{\hat{f}}_{A}(z)$ are continuous functions.

${ }^{5}$ For the case $y_{1}<y^{\prime}$, one should consider as domains: $0 \leq y \leq y_{1}, y_{1}<y \leq y^{\prime}$ and $y^{\prime}<y<y_{s}$; leading to a general solution $G_{A}\left(y, y^{\prime} ; p\right)$ whose expression differs slightly from eq. (3.8) due to the different definitions of the domains.

${ }^{6}$ Given the square root function, $f(z)=\sqrt{z}$, we will define the first Riemann sheet in the complex plane $z=|z| e^{i \varphi} \in \mathbb{C}$ as the one corresponding to $\varphi \in(-\pi, \pi]$, so that this function has a branch cut along the negative real axis. The second Riemann sheet is reached by shifting $\varphi \rightarrow \varphi+2 \pi$, i.e. it corresponds to $\varphi \in(\pi, 3 \pi]$. Then, the relation between the square root in the first, $f_{\mathrm{I}}(z)$, and second, $f_{\mathrm{II}}(z)$, Riemann sheets is $f_{\mathrm{II}}(z)=-f_{\mathrm{I}}(z)$, cf. e.g. ref. [45] and references therein.
} 
where only the behavior on the first variable $y$ is shown in the Green's functions, and $\Delta f(y) \equiv \lim _{\epsilon \rightarrow 0}(f(y+\epsilon)-f(y-\epsilon))$. In addition, we should impose regularity at the singularity $y=y_{s}$, i.e. $C_{1}^{I I I}=0$. This corresponds to outgoing wave boundary condition in Lorentzian AdS, which follows from the analytical continuation of the IR regular solution for Euclidean AdS [46], i.e. in conformal coordinates

$$
G_{A}\left(z, z^{\prime} ; p\right) \underset{z_{1} \ll z}{\simeq} e^{-\frac{1}{2} \Delta_{A}^{+} \rho z} \propto e^{-\sqrt{m_{g}^{2}-p^{2}} z}=e^{i \sqrt{-m_{g}^{2}+p^{2}} z},
$$

where in the last equality we have assumed that $p>m_{g}$, and adopted the prescription mentioned above. ${ }^{7}$ Then, all the integration constants are fixed. After conveniently defining the variables

$$
y_{\downarrow} \equiv \min \left(y, y^{\prime}\right), \quad y_{\uparrow} \equiv \max \left(y, y^{\prime}\right),
$$

and after implementing the boundary and matching conditions in the general solution of eq. (3.8) for $y^{\prime}<y_{1}$ (and in the equivalent general solution for $y_{1}<y^{\prime}$ ), one finds

$$
G_{A}\left(y, y^{\prime} ; p\right)=\left\{\begin{array}{cl}
\frac{\pi}{2 k} e^{k\left(y+y^{\prime}\right) \frac{\mathcal{P}\left(y_{\downarrow}\right) \mathcal{Z}\left(y_{\uparrow}\right)}{\Phi(p)}} & y_{\downarrow}, y_{\uparrow} \leq y_{1} \\
-\frac{2}{\rho} e^{k y_{\downarrow}}\left(k\left(y_{s}-y_{\uparrow}\right)\right)_{A}^{+} / 2 \frac{\mathcal{P}\left(y_{\downarrow}\right)}{\Phi(p)} & y_{\downarrow} \leq y_{1}<y_{\uparrow}, \\
\left(\frac{y_{s}-y_{\uparrow}}{y_{s}-y_{\downarrow}}\right)^{\Delta_{A}^{+} / 2} \delta_{A}^{-1} \cdot \frac{\mathcal{Q}\left(y_{\downarrow}\right)}{\Phi(p)} & y_{1}<y_{\downarrow}, y_{\uparrow}
\end{array}\right.
$$

a solution valid for $0 \leq y, y^{\prime}<y_{s}$. The functions $\Phi(p), \mathcal{P}(y), \mathcal{Z}(y)$ and $\mathcal{Q}(y)$ are defined as

$$
\begin{aligned}
& \Phi(p)=Y_{0}(p / k) \cdot J_{+}(p / \rho)-J_{0}(p / k) \cdot Y_{+}(p / \rho), \\
& \Psi(p)=Y_{0}(p / k) \cdot J_{-}(p / \rho)-J_{0}(p / k) \cdot Y_{-}(p / \rho), \\
& \mathcal{P}(y)=Y_{0}(p / k) \cdot J_{1}\left(e^{k y} p / k\right)-J_{0}(p / k) \cdot Y_{1}\left(e^{k y} p / k\right), \\
& \mathcal{Z}(y)=J_{+}(p / \rho) \cdot Y_{1}\left(e^{k y} p / k\right)-Y_{+}(p / \rho) \cdot J_{1}\left(e^{k y} p / k\right), \\
& \mathcal{Q}(y)=-\frac{k}{\rho^{2}} \frac{1}{k\left(y_{s}-y\right)}\left[\Phi(p)-\left(k\left(y_{s}-y\right)\right)^{\delta_{A}} \Psi(p)\right],
\end{aligned}
$$

where we define

$$
J_{ \pm}(p / \rho)=2 \frac{p}{\rho} J_{0}(p / \rho)+\Delta_{A}^{ \pm} J_{1}(p / \rho), \quad Y_{ \pm}(p / \rho)=2 \frac{p}{\rho} Y_{0}(p / \rho)+\Delta_{A}^{ \pm} Y_{1}(p / \rho) .
$$

Up to now we have not made any approximation. However, some of these functions can be slightly simplified by assuming $p \ll k$. In this case $J_{0}(p / k) \simeq 1+\mathcal{O}\left((p / k)^{2}\right)$ and $Y_{0}(p / k) \simeq \mathcal{K}+\mathcal{O}\left((p / k)^{2}\right)$ with

$$
\mathcal{K} \equiv \frac{2}{\pi}\left(\gamma_{E}-\log (2)+\log (p / \rho)-k y_{1}\right)
$$

Then, the approximate expressions of $\Phi(p), \Psi(p)$ and $\mathcal{P}(y)$ for $p \ll k$ turn out to be

$$
\begin{aligned}
& \Phi(p) \simeq \mathcal{K} \cdot J_{+}(p / \rho)-Y_{+}(p / \rho), \quad \Psi(p) \simeq \mathcal{K} \cdot J_{-}(p / \rho)-Y_{-}(p / \rho) \\
& \mathcal{P}(y) \simeq \mathcal{K} \cdot J_{1}\left(e^{k y} p / k\right)-Y_{1}\left(e^{k y} p / k\right)
\end{aligned}
$$

${ }^{7}$ Notice that we are not considering in eq. (3.11) the incoming wave $\propto e^{-i \sqrt{-m_{g}^{2}+p^{2}} z}$ as this is singular. 
When $y=y_{0}$ we can consider further simplifications in $\mathcal{P}(y)$ as well as in $\mathcal{Z}(y)$. While all the computations in this paper will be performed by using the exact Green's function given by eqs. (3.13)-(3.15), we will provide sometimes in the text approximate formulas to make the explicit expressions simpler. ${ }^{8}$

Let us study some of the properties of the Green's functions. In the limit $y \rightarrow y_{0}$ the Green's function can be written in the simplified form

$$
G_{A}\left(y_{0}, y^{\prime} ; p\right)=\left[e^{k y^{\prime}} \mathcal{Z}\left(y^{\prime}\right) \Theta\left(y_{1}-y^{\prime}\right)-\frac{4}{\pi} \frac{k}{\rho}\left(k\left(y_{s}-y^{\prime}\right)\right)^{\Delta_{A}^{+} / 2} \Theta\left(y^{\prime}-y_{1}\right)\right] \frac{1}{p} \frac{1}{\Phi(p)} .
$$

Notice that the Green's function (3.13) can be expressed as the product of two functions in the form $G_{A}\left(y, y^{\prime} ; p\right)=\mathcal{A}\left(y_{\downarrow}\right) \mathcal{B}\left(y_{\uparrow}\right)$, and this can also be written as

$$
\mathcal{A}\left(y_{\downarrow}\right) \mathcal{B}\left(y_{\uparrow}\right)=\mathcal{A}(y) \mathcal{B}\left(y^{\prime}\right) \Theta\left(y^{\prime}-y\right)+\mathcal{A}\left(y^{\prime}\right) \mathcal{B}(y) \Theta\left(y-y^{\prime}\right) .
$$

Then, it is clear that the Green's function is symmetric under the exchange of $y$ and $y^{\prime}$, i.e. it fulfills the property

$$
G_{A}\left(y, y^{\prime} ; p\right)=G_{A}\left(y^{\prime}, y ; p\right) .
$$

This property is not obvious from the EoM, eq. (3.7).

Another property is

$$
\operatorname{Im}\left(\mathcal{A}(y) \mathcal{B}\left(y^{\prime}\right)\right)=\operatorname{Im}\left(\mathcal{A}\left(y^{\prime}\right) \mathcal{B}(y)\right), \quad y, y^{\prime} \leq y_{1} \quad \text { or } \quad y, y^{\prime} \geq y_{1},
$$

for $p^{2}>0$, which follows from the explicit expressions of eq. (3.13), and taking into account the following relations

$$
\begin{aligned}
\left(\Delta_{A}^{ \pm}(p)\right)^{*} & =\Delta_{A}^{\mp}(p), \quad \Phi^{*}(p)=\Psi(p), \\
J_{ \pm}^{*}(p / \rho) & =J_{\mp}(p / \rho), \quad Y_{ \pm}^{*}(p / \rho)=Y_{\mp}(p / \rho), \quad\left(p^{2} \geq m_{g}^{2}\right),
\end{aligned}
$$

which are valid for time-like momenta. The properties given by eqs. (3.21) and (3.22) will be relevant for the study of the spectral functions in section 3.4.

\subsection{Brane-to-brane Green's functions}

Using the general result for $G_{A}\left(y, y^{\prime} ; p\right)$, one can obtain the particularly interesting cases of brane-to-brane Green's functions for gauge bosons. There are three relevant cases: $i$ ) UV-to-UV, ii) UV-to-IR, and iii) IR-to-IR Green's functions; and they are obtained by considering the limits

$$
G_{A}\left(y_{\alpha}, y_{\beta} ; p\right)=\lim _{\substack{y \rightarrow y_{\alpha} \\ y^{\prime} \rightarrow y_{\beta}}} G_{A}\left(y, y^{\prime} ; p\right)
$$

In particular, the UV-to-UV Green's function can be computed as well by using the holographic formalism, see ref. [13]. It is interesting and useful to provide the explicit analytical

\footnotetext{
${ }^{8}$ Notice that when considering momenta $p \sim \mathcal{O}(\rho)$, which will be the case throughout this paper, we are neglecting in eqs. (3.16)-(3.17) corrections of order $\mathcal{O}\left((\rho / k)^{2}\right)=\mathcal{O}\left(10^{-30}\right)$, so that the approximation made in these expressions turns out to be extremely good.
} 
expressions for the brane-to-brane Green's functions. These are

$$
\begin{aligned}
& G_{A}^{-1}\left(y_{0}, y_{0} ; p\right)=\frac{p \Phi(p)}{\mathcal{Z}\left(y_{0}\right)} \simeq-\frac{\pi p^{2}}{2 k} \cdot \frac{\Phi(p)}{J_{+}(p)} \\
& G_{A}^{-1}\left(y_{0}, y_{1} ; p\right)=-\frac{\pi}{4} \frac{\rho}{k} p \Phi(p) \\
& G_{A}^{-1}\left(y_{1}, y_{1} ; p\right)=-\frac{\rho^{2}}{2 k} \cdot \frac{\Phi(p)}{\mathcal{P}\left(y_{1}\right)} \simeq-\frac{\rho^{2}}{2 k} \cdot \frac{\Phi(p)}{\mathcal{K} \cdot J_{1}\left(\frac{p}{\rho}\right)-Y_{1}\left(\frac{p}{\rho}\right)},
\end{aligned}
$$

where in the second equality of eqs. (3.24) and (3.26) we have assumed $p \ll k$. All Green's functions include the zero-mode contribution which behaves as

$$
G_{A}^{0}=\frac{1}{y_{s} p^{2}}=\lim _{p \rightarrow 0} G_{A}\left(y, y^{\prime} ; p\right)
$$

which, after coupling to two fermions lines, with strength $g_{5}^{2}$, yields the usual 4D behavior $g_{4}^{2} / p^{2}$. Then, we can define Green's functions contributed only by the continuum KK modes, with the zero-mode contribution subtracted out, as

$$
\mathcal{G}_{A}\left(y, y^{\prime} ; p\right)=G_{A}\left(y, y^{\prime} ; p\right)-G_{A}^{0} .
$$

Note that while scale invariance is explicitly broken by the scales $\rho$ and $k$, it is possible to define rescaled Green's functions $\mathbb{G}_{A}\left(y, y^{\prime} ; p\right)=\mathcal{F}_{G} \cdot G_{A}\left(y, y^{\prime} ; p\right)$ that turn out to be dimensionless, and their dependence on momenta and scales is through dimensionless products and ratios $\mathbb{G}_{A}\left(k y, k y^{\prime} ; p / \rho, \rho / k\right)$ (cf. ref. [13]). The required scaling factor

$$
\mathcal{F}_{G}=\rho\left(\frac{\rho}{k}\right)^{a}\left(k y_{s}\right)^{b}
$$

where $a$ and $b$ are real numbers, is independent of the momentum $p$. We will be interested in the effect on the brane-to-brane Green's functions of a change of the scale $\rho$. To this end, we will consider a rescaling of the form

$$
p \rightarrow p^{\prime}=c p, \quad \rho \rightarrow \rho^{\prime}=c \rho \quad \text { and } \quad k \rightarrow k^{\prime}=\bar{c} k,
$$

with $c \neq \bar{c}$. While the ratio $p / \rho$ is not affected by the rescaling, one has $\rho / k \rightarrow(c / \bar{c}) \cdot \rho / k$. Using that $k y_{s}=1-\log (\rho / k)$, cf. eqs. (2.15)-(2.16), one can see that eq. (3.30) implies a shift of $k y_{s}\left(\right.$ or $\left.A\left(y_{1}\right)\right)$, i.e. $k y_{s} \rightarrow k y_{s}-\log (c / \bar{c})$. When considering $a=1$ in eq. (3.29), the dimensionless brane-to-brane Green's functions $\mathbb{G}_{A}=\mathbb{G}_{A}(p / \rho, \rho / k)$ turn out to have a smooth logarithmic dependence on $\rho / k$, so that the effect of the scaling parameters $c$ and $\bar{c}$ is also logarithmic. In addition, it is possible to choose the parameter $b$ in eq. (3.29) so that the corresponding scaling factor $\mathcal{F}_{G}$ removes the dominant dependence in $\log (\rho / k)$ of the respective Green's function, making it almost invariant under shifts of $k y_{s}$. While the factor that makes $G_{A}^{0}$ invariant is $\mathcal{F}_{G}=\frac{\rho^{2}}{k}\left(k y_{s}\right)$, the brane-to-brane Green's functions with the zero-mode subtracted out $\mathcal{G}_{A}\left(y_{\alpha}, y_{\beta} ; p\right)$ will be approximately invariant under shifts of $k y_{s}$ when multiplying them by the factors $\mathcal{F}_{\alpha \beta}$, where

$$
\mathcal{F}_{00}=\frac{\rho^{2}}{k}\left(k y_{s}\right)^{2}, \quad \mathcal{F}_{01}=\frac{\rho^{2}}{k}\left(k y_{s}\right), \quad \mathcal{F}_{11}=\frac{\rho^{2}}{k} .
$$

These factors will be used in the rest of the manuscript, in particular in section 3.4 for the spectral functions, as well as in section 4 and appendix A. 

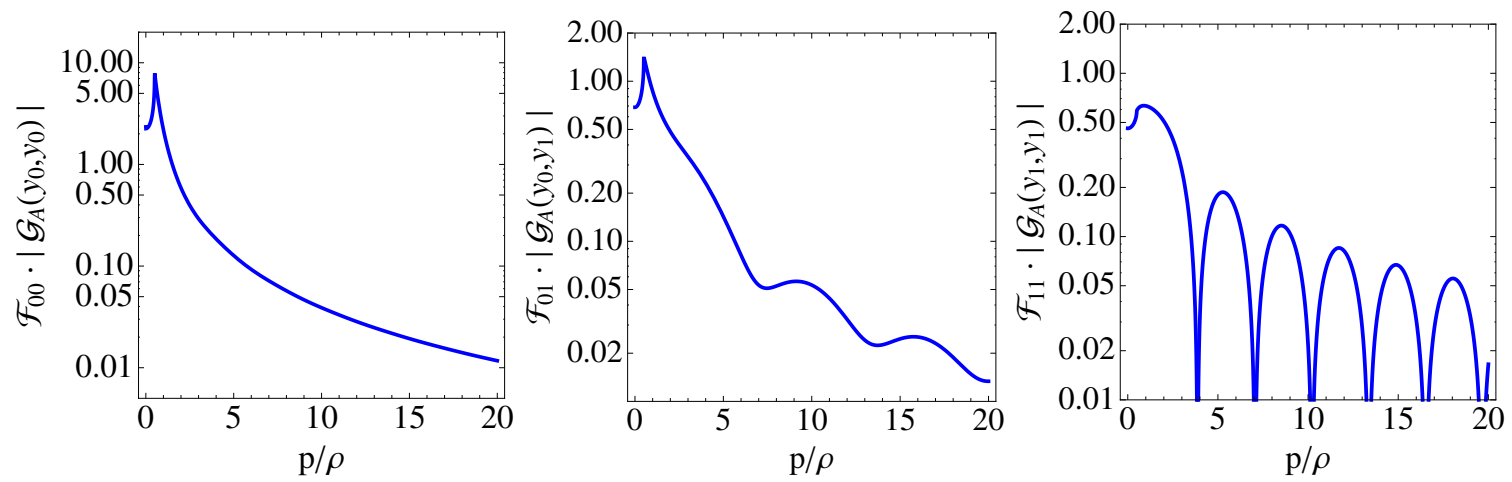

Figure 2. Plots of the rescaled Green's functions $\mathcal{F}_{00} \cdot\left|\mathcal{G}_{A}\left(y_{0}, y_{0} ; p\right)\right|$ (left panel), $\mathcal{F}_{01} \cdot\left|\mathcal{G}_{A}\left(y_{0}, y_{1} ; p\right)\right|$ (middle panel), and $\mathcal{F}_{11} \cdot\left|\mathcal{G}_{A}\left(y_{1}, y_{1} ; p\right)\right|$ (right panel) as functions of $p / \rho$. We have used $A_{1}=35$ in all panels and assume time-like momenta $p^{2}>0$.
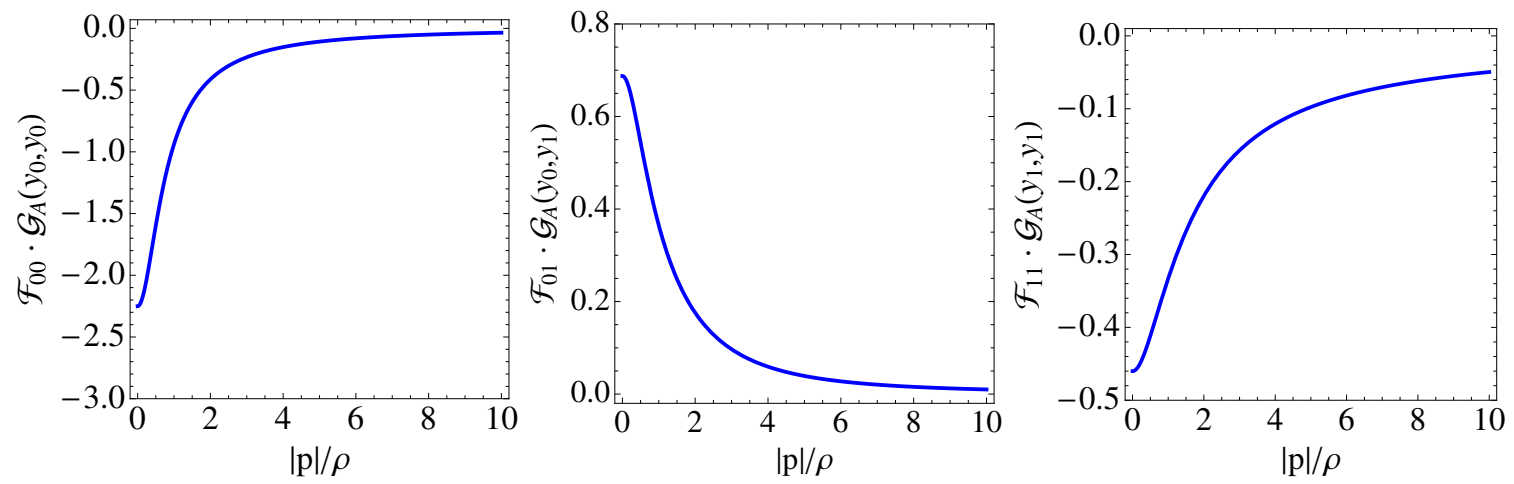

Figure 3. Plots of $\mathcal{F}_{00} \cdot \mathcal{G}_{A}\left(y_{0}, y_{0} ;|p|\right)$ (left panel), $\mathcal{F}_{01} \cdot \mathcal{G}_{A}\left(y_{0}, y_{1} ;|p|\right)$ (middle panel) and $\mathcal{F}_{11}$. $\mathcal{G}_{A}\left(y_{1}, y_{1} ;|p|\right)$ (right panel) as a function of $|p| / \rho$. We have used $A_{1}=35$ in all panels and assume space-like momenta $p^{2}<0$.

We plot, in figure $2,\left|\mathcal{G}_{A}\left(y_{0}, y_{0} ; p\right)\right|$ (left panel), $\left|\mathcal{G}_{A}\left(y_{0}, y_{1} ; p\right)\right|$ (middle panel), and $\left|\mathcal{G}_{A}\left(y_{1}, y_{1} ; p\right)\right|$ (right panel), normalized by the factors $\mathcal{F}_{\alpha \beta}$ of eq. (3.31), as functions of $p / \rho$, for time-like momenta $p^{2}>0$. For space-like momenta $p^{2}<0$ the Green's functions are purely real. In figure 3 we plot the Green's functions $\mathcal{G}_{A}\left(y_{0}, y_{0} ;|p|\right), \mathcal{G}_{A}\left(y_{0}, y_{1} ;|p|\right)$ and $\mathcal{G}_{A}\left(y_{1}, y_{1} ;|p|\right)$, normalized by $\mathcal{F}_{\alpha \beta}$, as functions of $|p| / \rho$, for space-like momenta $p^{2}<0$.

It is also interesting to provide the limits $p \ll \rho$ of the Green's functions. This leads to the following Taylor series expansions

$$
\begin{aligned}
& G_{A}^{-1}\left(y_{0}, y_{0} ; p\right) \underset{p \ll \rho}{\simeq} y_{s} p^{2}+\frac{9}{4 k} \frac{p^{4}}{\rho^{2}}+\mathcal{O}\left(p^{6}\right), \\
& G_{A}^{-1}\left(y_{0}, y_{1} ; p\right) \underset{p \ll \rho}{\simeq} y_{s} p^{2}+\frac{\left(9-3 k y_{s}\right)}{4 k} \frac{p^{4}}{\rho^{2}}+\mathcal{O}\left(p^{6}\right), \\
& G_{A}^{-1}\left(y_{1}, y_{1} ; p\right) \underset{p \ll \rho}{\simeq} y_{s} p^{2}+\frac{\left(9+2 k y_{s}\left(-3+k y_{s}\right)\right)}{4 k} \frac{p^{4}}{\rho^{2}} \\
& +\frac{\left(113+4 k y_{s}\left[63+2 k y_{s}\left(-19+4 k y_{s}\right)\right]\right)}{128 k} \frac{p^{6}}{\rho^{4}}+\mathcal{O}\left(p^{8}\right) .
\end{aligned}
$$


The $p^{2}$ behavior is valid for any Green's function, i.e. $G_{A}^{-1}\left(y, y^{\prime} ; p\right) \underset{p \ll \rho}{\simeq} y_{s} p^{2}+\cdots .9$ We keep terms up to $\mathcal{O}\left(p^{6}\right)$ in the IR-to-IR Green's function as these will be needed in the computation of electroweak precision observables of section 5 .

Finally, the behavior in the regime $\rho \ll p$ (and $p \ll k$ ) is, for time-like momenta, $p^{2}>0$

$$
\begin{aligned}
& G_{A}^{-1}\left(y_{0}, y_{0} ; p\right) \underset{\rho \ll p}{\simeq}\left(k y_{1}-\log \left(\frac{p}{\rho}\right)+i \frac{\pi}{2}\right)\left(\frac{p}{\rho}\right)^{2} \frac{\rho^{2}}{k}, \\
& G_{A}^{-1}\left(y_{0}, y_{1} ; p\right) \underset{\rho \ll p}{\simeq} \sqrt{\frac{2}{\pi}} e^{-i(p / \rho-\pi / 4)}\left(k y_{1}-\log \left(\frac{p}{\rho}\right)+i \frac{\pi}{2}\right)\left(\frac{p}{\rho}\right)^{3 / 2} \frac{\rho^{2}}{k}, \\
& G_{A}^{-1}\left(y_{1}, y_{1} ; p\right) \underset{\rho \ll p}{\simeq} \frac{e^{-i(p / \rho-\pi / 4)}}{\cos \left(\frac{p}{\rho}+\frac{\pi}{4}\right)}\left(\frac{p}{\rho}\right) \frac{\rho^{2}}{k},
\end{aligned}
$$

and for space-like momenta $p^{2}<0, p \equiv i|p|$,

$$
\begin{aligned}
& G_{A}^{-1}\left(y_{0}, y_{0} ;|p|\right) \underset{\rho \ll|p|}{\simeq}\left(\log \left(\frac{|p|}{\rho}\right)-k y_{1}\right)\left|\frac{p}{\rho}\right|^{2} \frac{\rho^{2}}{k}, \\
& G_{A}^{-1}\left(y_{0}, y_{1} ;|p|\right) \underset{\rho \ll|p|}{\simeq} \sqrt{\frac{2}{\pi}} e^{|p| / \rho}\left(\log \left(\frac{|p|}{\rho}\right)-k y_{1}\right)\left|\frac{p}{\rho}\right|^{3 / 2} \frac{\rho^{2}}{k}, \\
& G_{A}^{-1}\left(y_{1}, y_{1} ;|p|\right) \underset{\rho \ll|p|}{\simeq}-2\left|\frac{p}{\rho}\right| \frac{\rho^{2}}{k} .
\end{aligned}
$$

Notice that for space-like momenta the Green's function $G_{A}\left(y_{0}, y_{1} ;|p|\right)$ goes exponentially to zero as $e^{-|p| / \rho}$ for $|p| \gg \rho$, a property which was recently noticed in ref. [47]. The general asymptotic behavior for the Green's function $G_{A}\left(z_{0}, z^{\prime} ;|p|\right)$, with $z^{\prime}<z_{1}$, is $\sim$ $\exp \left(-z^{\prime}|p| / z_{1} \rho\right)$

\subsection{Green's functions in the complex plane and resonances}

Although the spectrum of excitations is a continuum, starting from the mass gap $m_{g}=\rho / 2$, which is characteristic of a conformal theory, as the conformal invariance is explicitly (spontaneously) broken by the UV (IR) brane it is worth exploring the structure of the Green's functions in the complex $s \equiv p^{2}$ plane, with

$$
s \equiv M^{2}-i M \Gamma=M^{2}(1-i r), \quad r \equiv \Gamma / M,
$$

as it is well known in Quantum Field Theory that resonances with mass $M$ and decay width $\Gamma$ are associated to the presence of poles in the unphysical Riemann sheet. Needless to say, in ordinary Quantum Field Theory, the presence of poles in the complex plane are associated to production processes corresponding to decays of the resonance into other particles of mass $m$, for energies above the threshold $s>4 m^{2}$. Nevertheless our Green's functions, even considered at the classical level, have an imaginary part, unrelated to any decay process, similarly to the case of unparticles. Still exploring the complex $s$ plane

\footnotetext{
${ }^{9}$ Notice that the rescaled Green's functions behave as $\mathbb{G}_{A}^{-1}\left(y, y^{\prime} ; p\right) \underset{p \ll \rho}{\simeq}(p / \rho)^{2}+\cdots$, and they turn out to be functions of $p / \rho$ with "power-like" corrections in $k y_{s}=1-\log (\rho / k)$.
} 
is worth given that, as we stated above, conformal invariance is broken, which makes a fundamental difference with respect to the case of unparticles.

Let us study the possible existence of poles of the Green's functions $G_{A}\left(y, y^{\prime} ; s\right)$ in the complex $s$-plane. As the origin of the non-vanishing imaginary part of Green's functions is the threshold function $\delta_{A}(s)=\sqrt{1-4 s / \rho^{2}}$ which has two Riemann sheets, similar to the threshold function of the decay into two particles, in order to perform an analysis of the resonances, one should compute the Green's functions in the second Riemann sheet of the square root function. It can be easily seen that a change from the first (I) to the second (II) Riemann sheet is equivalent to the replacement $\delta_{A}^{\mathrm{II}}(s) \rightarrow-\delta_{A}^{\mathrm{I}}(s)=-\delta_{A}(s)$, i.e.

$$
\delta_{A}^{\mathrm{II}}(s)=-\sqrt{1-4 s / \rho^{2}},
$$

where the square root in this formula is the one in the first Riemann sheet. Let us point out that the function $\delta_{A}(s)$, and then the Green's functions $G_{A}\left(y, y^{\prime} ; s\right)$, have a branch cut from the mass gap $s=\rho^{2} / 4$ to infinity along the real axis with the first Riemann sheet corresponding to $\varphi \in[0,2 \pi)$, where $\varphi$ is defined as $s-\rho^{2} / 4=\left|s-\rho^{2} / 4\right| e^{i \varphi}$. The second Riemann sheet corresponds to $\varphi \in[2 \pi, 4 \pi)$. The Green's functions are continuous when changing from the first to the second Riemann sheets, but there appears a discontinuity at the branch cut if one approaches it using the same Riemann sheet. This discontinuity is accounted by the spectral function.

From eqs. (3.24)-(3.26), one realizes that the possible poles (excluding the zero-mode) should appear as zeros of the function $\Phi(p)$. Following this idea, we display in figure 4 a contour plot of $\log _{10}|\Phi(p)|$ computed in the second Riemann sheet. For convenience, we have expressed the squared complex momenta $s$ in the plane $M / \rho$ and $r$ given in eq. (3.41). One can see from the left panel of figure 4 that there appears an intriguing structure of zeros of the function $\Phi(p)$ in the $s$ plane. In the right panel of figure 4 we plot the function $|\Phi|$ along an interpolating curve connecting the zeros of $\Phi(p)$ (the red points in the left panel of figure 4), as a function of $M / \rho$. We can check that the $\Phi$ function indeed vanishes at those points.

All resonances appear for both positive and negative values of $\Gamma$. The latter are unphysical shadow poles, required by Hermitian analyticity [27, 48]. The lightest resonances appear at the values

$$
(M / \rho, r)=(2.42,2.87),(6.03,1.12),(9.37,0.768),(12.64,0.601),(15.87,0.500), \cdots .
$$

The values of $M / \rho$ follow a pattern similar to the KK modes in the RS model, for which the eigenvalues $M_{n} / \rho$ are close to the zeros of the $J_{0}\left(M_{n} / \rho\right)$ function. However, contrary to the RS model, the resonances in the gapped continuum model have a finite width. This width increases slowly with energy, but the relative width $\Gamma / M$ decreases, so that the resonances tend to a distribution closer to Dirac delta functions at high energies.

We can study analytically the location of these zeros in the following way. If one performs an expansion of the function $\Phi(p)$ at large momentum $\rho \ll|p|(|p| \ll k)$ one finds

$$
\Phi(p) \underset{\rho \ll|p|}{\simeq} \frac{e^{-i(p / \rho+\pi / 4)}}{\sqrt{2 \pi^{3}}}\left[e^{i 2 p / \rho}-8 i\left(\frac{p}{\rho}\right)^{2}\right] \log \left(\frac{p}{k}\right)\left(\frac{\rho}{p}\right)^{3 / 2}, \quad \operatorname{Im}\left((p / \rho)^{2}\right)<0 .
$$



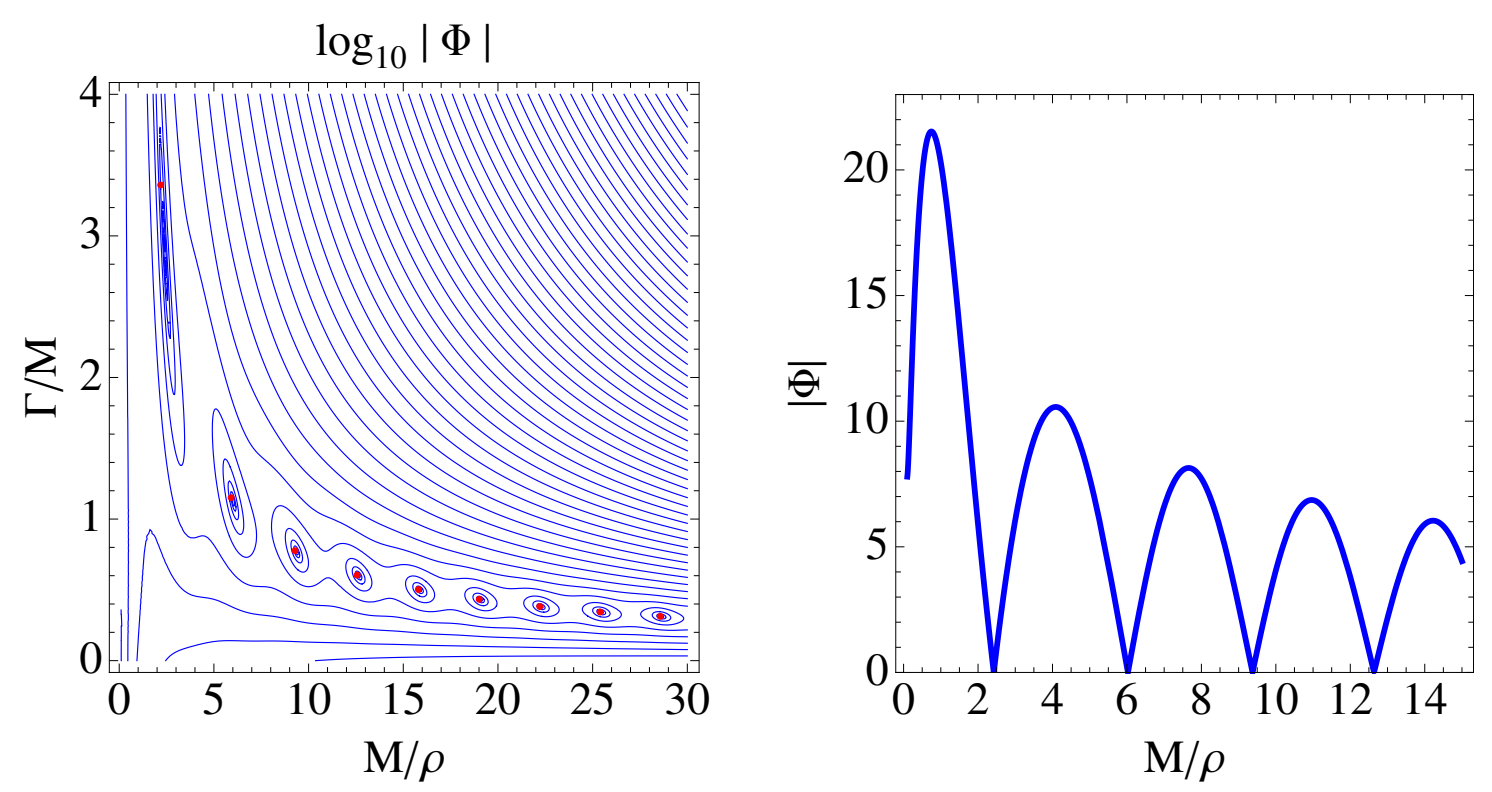

Figure 4. Left panel: contour plot of $\log _{10}|\Phi(p)|$ in the plane $(M / \rho, \Gamma / M)$. The contour lines form small circles around the zeros of $\Phi(p)$. The (red) dots stand for the positions of the zeros of $\Phi(p)$ as given by the analytical formula of eq. (3.46). Right panel: plot of $|\Phi(p)|$ along an interpolating curve connecting the zeros of $\Phi(p)$ appearing in the left panel. We have used the variables $M / \rho$ and $r \equiv \Gamma / M$ defined in eq. (3.41). We have considered $A_{1}=35$.

Then, the zeros of $\Phi(p)$ correspond to the solutions of the equation

$$
e^{i 2 p / \rho}=8 i\left(\frac{p}{\rho}\right)^{2}
$$

which turn out to be

$$
\frac{s}{\rho^{2}}=-\mathcal{W}_{n}\left[\frac{\varepsilon}{4}(1+i)\right]^{2}, \quad \varepsilon= \pm 1, \quad n=-1,-2,-3, \cdots
$$

Here $\mathcal{W}_{n}$ is the $n$-th branch of the Lambert function. ${ }^{10}$ We display as red dots in figure 4 (left) the results of eq. (3.46). Moving from lighter to heavier resonances corresponds to taking $(n, \varepsilon)=(-1,+1),(-1,-1),(-2,+1),(-2,-1), \cdots$, in this order. Note the close agreement of the analytical results with the true zeros of $\Phi(p)$, even for the lightest resonances: the relative error for the values of $M / \rho$ and $\Gamma / M$ decreases with $M / \rho$, and it is $\lesssim 2 \%$ except for the lightest resonance which is $\sim 15 \%$.

We display in figure 5 contour plots in the plane $(M / \rho, \Gamma / M)$ of the (common logarithm of the absolute value of the) brane-to-brane Green's functions computed in the second Riemann sheet. All Green's functions $G_{A}\left(y_{\alpha}, y_{\beta} ; s\right)$ exhibit poles in the complex $s$-plane located at the corresponding zeros of the function $\Phi(p)$. In the case of $G_{A}\left(y_{0}, y_{0}\right)$, for each pole of the Green's function there appears a zero close to it. The function $J_{+}(p)$ in the

\footnotetext{
${ }^{10}$ The Lambert function is the solution of the equation $z=\mathcal{W}(z) e^{\mathcal{W}(z)}$. In addition to the principal branch $n=0$, there are other infinite branches denoted by $\mathcal{W}_{n}(z)$ for integer $n$.
} 

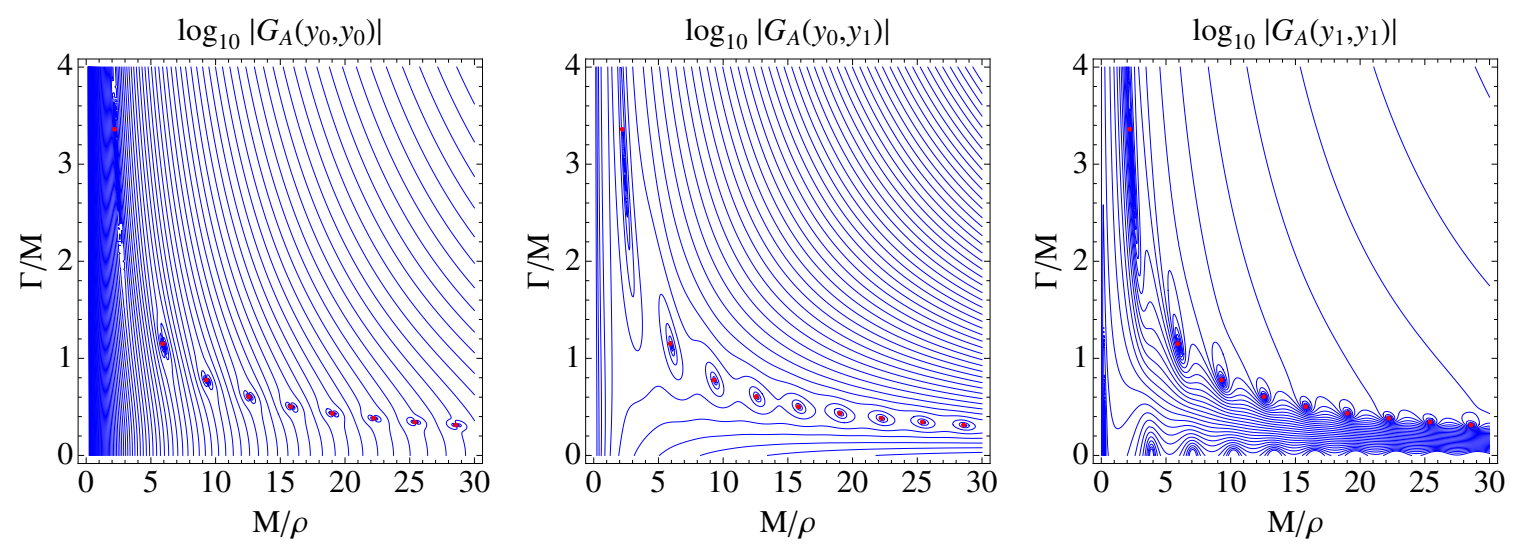

Figure 5. Contour plot in the plane $(M / \rho, \Gamma / M)$ of the common logarithm of the absolute value of the Green's functions $\log _{10}\left|G_{A}\left(y_{0}, y_{0}\right)\right|$ (left panel), $\log _{10}\left|G_{A}\left(y_{0}, y_{1}\right)\right|$ (middle panel) and $\log _{10}\left|G_{A}\left(y_{1}, y_{1}\right)\right|$ (right panel). The (red) dots stand for the positions of the poles of the Green's functions as predicted by the analytical formula of eq. (3.46). The contour lines form small circles around the poles of $G_{A}\left(y_{\alpha}, y_{\beta}\right)$ (those circles with red dots), or circles around the zeros of $G_{A}\left(y_{\alpha}, y_{\beta}\right)$ (those circles with no red dots). We have considered $A_{1}=35$.

numerator of $G_{A}\left(y_{0}, y_{0}\right)$, in eq. (3.24), behaves, for large momentum, as

$$
J_{+}(p) \underset{\rho \ll|p|}{\simeq} \frac{e^{-i(p / \rho+\pi / 4)}}{\sqrt{8 \pi}}\left[e^{i 2 p / \rho}-8 i\left(\frac{p}{\rho}\right)^{2}\right]\left(\frac{\rho}{p}\right)^{3 / 2}, \quad \operatorname{Im}\left((p / \rho)^{2}\right)<0 .
$$

From a comparison with eq. (3.44), we conclude that the zeros of $G_{A}\left(y_{0}, y_{0}\right)$ turn out to be very close to its poles, and their residues approximately cancel. In order to quantitatively characterize the difference between the positions of these zeros and poles, we have displayed in figure 6 the relative difference in the plane $(M, \Gamma)$ of the location of the zeros of $\Phi(p)$ and of $J_{+}(p)$ in the second Riemann sheet, with $s_{\Phi} \equiv M_{\Phi}^{2}-i M_{\Phi} \Gamma_{\Phi}$ and $s_{J_{+}} \equiv M_{J_{+}}^{2}-i M_{J_{+}} \Gamma_{J_{+}}$, respectively. This difference is $\lesssim 3 \%$, and rapidly decreases for heavier resonances. Finally, let us notice that the Green's function $G_{A}\left(y_{0}, y_{1}\right)$ does not have any zero, while $G_{A}\left(y_{1}, y_{1}\right)$ has also zeros located in the real axis, corresponding to zeros in the denominator of eq. (3.26). Subsequently there is no suppression of the pole residues in these cases.

It is interesting to realize that the poles of the Green's function in the real axis of the complex $s$ plane correspond to eigenvalues of the EoM of the fluctuations (3.2), a property that can be checked as follows. The wave function $f_{A}(y)$ is subject to the following boundary condition in the UV brane and jumping conditions in the IR brane

$$
\left.C_{\mathrm{UV}}(p) \equiv \frac{\partial_{y} f_{A}(y)}{f_{A}(y)}\right|_{y=0}=0, \quad \Delta f_{A}\left(y_{1}\right)=0, \quad \Delta\left(\partial_{y} f_{A}\right)\left(y_{1}\right)=0
$$

In addition, for states with mass below the mass gap (the zero mode) regularity should be imposed at the singularity $y=y_{s}$ which implies $f_{A}(y) \underset{y \rightarrow y_{s}}{\simeq}\left(y_{s}-y\right)^{\frac{1}{2} \Delta_{A}^{+}}$, an IR behavior that can be written more explicitly as

$$
f_{A}(z) \underset{z_{1} \ll z}{\propto} e^{-\sqrt{m_{g}^{2}-p^{2}} z} \Theta\left(m_{g}^{2}-p^{2}\right)+e^{i \sqrt{-m_{g}^{2}+p^{2}} z} \Theta\left(p^{2}-m_{g}^{2}\right) .
$$




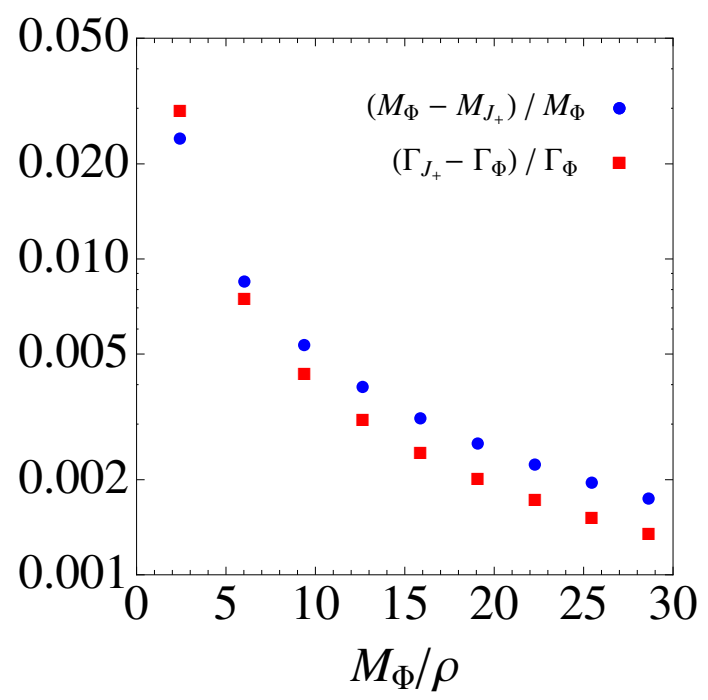

Figure 6. Relative difference, in the plane $(M, \Gamma)$, between the positions of the zeros of $\Phi(p)$ and of $J_{+}(p)$ computed in the second Riemann sheet, corresponding to the poles and zeros of the Green's function $G_{A}\left(y_{0}, y_{0}\right)$, respectively. These zeros are denoted by $\left(M_{\Phi}, \Gamma_{\Phi}\right)$ and $\left(M_{J_{+}}, \Gamma_{J_{+}}\right)$, respectively.

However, regularity in the IR singularity should not be imposed for states with mass above the mass gap. The general solution of the EoM of the fluctuations (3.2) contains four integration constants $C_{i}^{I, I I}(i=1,2)$, i.e. two constants per region: i) Region I: $0<y<y_{1}$, and ii) Region II: $y_{1}<y<y_{s}$. The jumping conditions in the IR brane fix two of the constants.

Let us first discuss the eigenvalue problem below the mass gap $\left(p<m_{g}\right)$. In this case, the regularity condition at $y=y_{s}$ fixes one of the integration constants, as the solution with + and - in the first and second exponents of eq. (3.49), respectively, is absent. The remaining integration constant can only be fixed by normalization of the wave function. Finally, the UV boundary condition is fulfilled only for certain values of the momentum. From an explicit computation of $C_{\mathrm{UV}}(p)$, it turns out that

$$
C_{\mathrm{UV}}(p)=G_{A}^{-1}\left(y_{0}, y_{0} ; p\right)
$$

where the explicit expression of the UV-to-UV Green's function is given by eq. (3.24). Then, we conclude that the values of the momenta $p$ fulfilling the UV boundary condition $\left(C_{\mathrm{UV}}(p)=0\right)$ correspond exactly to the poles of the Green's function, in our case to the zero mode $\left(p^{2}=0\right)$. Let us point out that only the zero mode (and not the resonances discussed in this section) corresponds to a genuine single bound state, and then to the solution of an eigenvalue problem for a Hermitian Hamiltonian $\left(p^{2} \in \mathbb{R}\right) .{ }^{11}$

\footnotetext{
${ }^{11}$ An analysis similar to the one presented above was also performed for the radion field within the LDM in ref. [15]. In this case, the condition (3.50) (with $G_{A}$ replaced by the Green's function of the radion) was also obtained, and it correctly predicted the mass of the radion corresponding to a single bound state below the mass gap.
} 

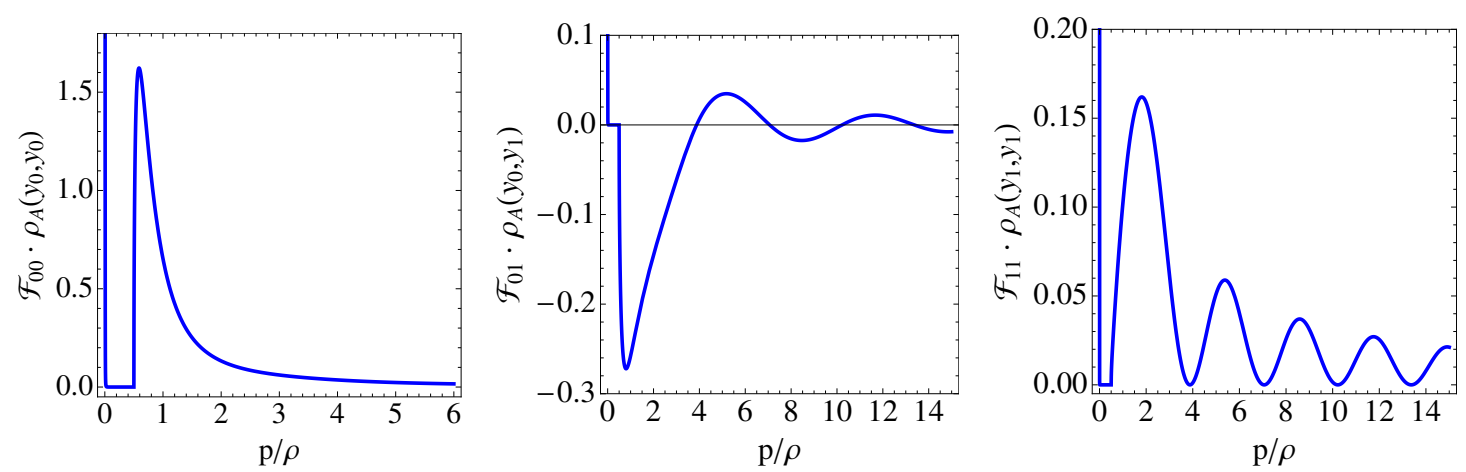

Figure 7. Rescaled spectral functions $\mathcal{F}_{00} \cdot \rho_{A}\left(y_{0}, y_{0} ; p\right)$ (left panel), $\mathcal{F}_{01} \cdot \rho_{A}\left(y_{0}, y_{1} ; p\right)$ (middle panel) and $\mathcal{F}_{11} \cdot \rho_{A}\left(y_{1}, y_{1} ; p\right)$ (right panel) as a function of $p / \rho$, for a continuum gauge boson. We have used $A_{1}=35$ in all panels and assume time-like momenta $p^{2}>0$.

Regarding the states with mass above the mass gap $\left(p>m_{g}\right)$, the three conditions in eq. (3.48) fix three of the integration constants. As in the case of the bound state, the remaining integration constant can only be fixed by normalization of the wave function in the continuum, something that can be done, for instance, as $\left\langle f_{p} \mid f_{p^{\prime}}\right\rangle=\delta\left(p^{2}-p^{\prime 2}\right)$. It is precisely the absence of the regularity condition at $y=y_{s}$ which gives rise to a continuum spectrum, analogous to scattering states in quantum mechanics.

\subsection{Spectral functions}

For time-like momenta, $p^{2}>0$, all Green's functions have imaginary contributions for values of $p>m_{g}=\rho / 2$, which is not associated to a particle threshold decay, an intrinsic property of e.g. unparticle theories. In this way we can define the corresponding spectral functions as

$$
\rho_{A}\left(y, y^{\prime} ; s\right) \equiv-\frac{1}{\pi} \operatorname{Im} G_{A}\left(y, y^{\prime} ; s+i \epsilon\right), \quad s \equiv p^{2} .
$$

In figure 7 we show the spectral functions $\rho_{A}\left(y_{0}, y_{0} ; p\right), \rho_{A}\left(y_{0}, y_{1} ; p\right)$ and $\rho_{A}\left(y_{1}, y_{1} ; p\right)$ as functions of $p / \rho$ where the prefactors, defined by eq. (3.31), make them approximately invariant for $p>0$ under a rescaling of the form of eq. (3.30), i.e. under shifts of the value of $k y_{s}$ [13]. By using the identity

$$
\lim _{\epsilon \rightarrow 0^{+}} \frac{1}{x+i \epsilon}=\mathcal{P} \frac{1}{x}-i \pi \delta(x),
$$

one can see that the small $p$ behavior of the Green's functions provided in section 3.2 implies the existence of a Dirac delta behavior in the spectral functions at $p=0$,

$$
\rho_{A}\left(y, y^{\prime} ; s\right)=\frac{1}{y_{s}} \delta(s)+\cdots .
$$

This delta function appears in all the spectral functions of figure 7 .

Notice that while the spectral functions $\rho_{A}\left(y_{0}, y_{0}\right)$ and $\rho_{A}\left(y_{1}, y_{1}\right)$ are positive definite (as they are $4 \mathrm{D}$ spectral functions in the corresponding $4 \mathrm{D}$ branes), the UV-to-IR brane spectral function $\rho_{A}\left(y_{0}, y_{1}\right)$ is not, a fact that challenges the physical interpretation of the 
spectral function in $4 \mathrm{D}$ Quantum Field Theory, as it is positive definite by its probabilistic interpretation. This apparent contradiction was already noticed and addressed for the graviton field in the context of LDM, cf. ref. [15]. Following similar ideas, we will briefly explain below how the positivity of the spectral function in our theory is understood.

From the $4 \mathrm{D}$ point of view, the spectral function $\rho_{A}\left(y, y^{\prime} ; s\right)$ can be considered as the matrix element $\left(y, y^{\prime}\right)$ of an operator $\hat{\rho}_{A}$, i.e. ${ }^{12}$

$$
\left(\hat{\rho}_{A}\right)_{y}^{y^{\prime}} \equiv \rho_{A}\left(y, y^{\prime} ; s\right) \text {. }
$$

This operator acts on the infinite dimensional space parametrized by the coordinate $y$. We can similarly define for the Green's functions $G_{A}\left(y, y^{\prime} ; s\right)$ the operator $\hat{G}_{A}$ such that

$$
\hat{\rho}_{A}=-\frac{1}{\pi} \operatorname{Im} \hat{G}_{A}, \quad \text { where } \quad \operatorname{Im} \hat{G}_{A}=\frac{1}{2 i}\left(\hat{G}_{A}-\hat{G}_{A}^{\dagger}\right) .
$$

Let us clarify at this point that it is expected that the operator $\hat{\rho}_{A}$ is positive semidefinite, but this does not imply that every matrix element is positive semidefinite. The elements of $\hat{\rho}_{A}$ form an infinite (continuous) dimensional matrix. Using the explicit expressions for the Green's function given by eq. (3.13), and taking into account the properties of eqs. (3.21) and (3.22), it is possible to check that the determinant of any $2 \times 2$ submatrix is vanishing, i.e.

$$
\left(\hat{\rho}_{A}\right)_{2 \times 2}=\left(\begin{array}{cc}
\left(\hat{\rho}_{A}\right)_{y}^{y} & \left(\hat{\rho}_{A}\right)_{y^{\prime}}^{y} \\
\left(\hat{\rho}_{A}\right)_{y}^{y^{\prime}} & \left(\hat{\rho}_{A}\right)_{y^{\prime}}^{y^{\prime}}
\end{array}\right) \Longrightarrow \operatorname{det}\left(\hat{\rho}_{A}\right)_{2 \times 2}=\left(\hat{\rho}_{A}\right)_{y}^{y}\left(\hat{\rho}_{A}\right)_{y^{\prime}}^{y^{\prime}}-\left(\hat{\rho}_{A}\right)_{y^{\prime}}^{y}\left(\hat{\rho}_{A}\right)_{y}^{y^{\prime}}=0 .
$$

This property, together with $\left(\hat{\rho}_{A}\right)_{y}^{y^{\prime}}=\left(\hat{\rho}_{A}\right)_{y^{\prime}}^{y}$, cf. eq. (3.20), implies that the matrix $\hat{\rho}_{A}$ turns out to have a factorizable form, i.e. any matrix element can be written in the form

$$
\left(\hat{\rho}_{A}\right)_{y^{\prime}}^{y}=\rho_{y} \rho_{y^{\prime}} \quad \text { where } \quad \rho_{y}=\sqrt{\left(\hat{\rho}_{A}\right)_{y}^{y}} .
$$

Given this factorization property, it turns out that the operator $\hat{\rho}_{A}$ is positive semidefinite, and all its eigenvalues are zero except one $\lambda(p)$, which is given by the trace of the matrix, i.e.

$$
\lambda(p)=\operatorname{tr} \hat{\rho}_{A}=\int_{0}^{y_{s}} d y \rho_{A}(y, y ; p) .
$$

In particular, note that $\left(\hat{\rho}_{A}\right)_{y}^{y}=\rho_{y}^{2} \geq 0$ implies that $\lambda(p) \geq 0 .{ }^{13}$

In order to perform this integral, let us split it into two domains,

$$
\lambda(p)=\lambda_{01}(p)+\lambda_{1 s}(p),
$$

where

$$
\lambda_{01}(p) \equiv \int_{0}^{y_{1}} d y \rho_{A}(y, y ; p), \quad \lambda_{1 s}(p) \equiv \int_{y_{1}}^{y_{s}} d y \rho_{A}(y, y ; p) .
$$

\footnotetext{
${ }^{12}$ We could also use a Dirac notation for the matrix element, $\rho_{A}\left(y, y^{\prime} ; s\right)=\left\langle y\left|\hat{\rho}_{A}\right| y^{\prime}\right\rangle$. We thank Prof. L.L. Salcedo for a private communication on the meaning of the spectral operator and its matrix elements.

${ }^{13}$ Notice that a symmetric matrix is positive semidefinite if and only if all its eigenvalues are non-negative.
} 


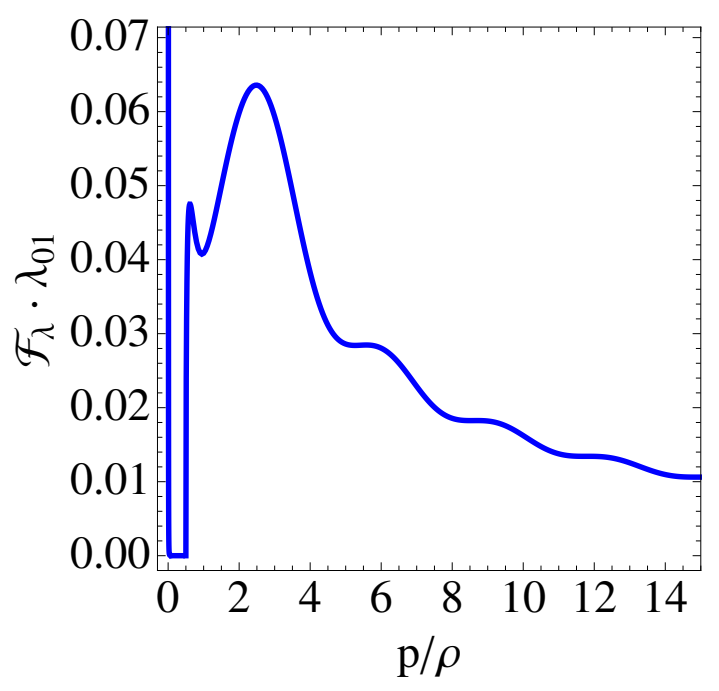

Figure 8. The UV-to-IR brane contribution to the rescaled $4 \mathrm{D}$ spectral function: $\mathcal{F}_{\lambda} \cdot \lambda_{01}(p)$. We have used $A_{1}=35$ and assume time-like momenta $p^{2}>0$.

The integral of $\lambda_{01}$ can easily be performed, and the result is plotted in figure 8 . The prefactor, defined as

$$
\mathcal{F}_{\lambda}=\rho^{2},
$$

makes it almost invariant under shifts of $k y_{s}$. Notice the appearance of softened peaks, at values $p / \rho \simeq n \pi$, i.e. at the positions of the resonances obtained in section 3.3. There is also a Dirac delta behavior at $p=0$. Focusing now on $\lambda_{1 s}$, it turns out to be divergent due to the term $\propto 1 /\left(y_{s}-y\right)$ in eq. (3.14), so it needs to be regularized. We will do it by introducing the cutoff $\bar{\epsilon}$ in the integral, so integrating up to $k y_{s}-\bar{\epsilon}$. The integral will then be dominated by its value at $k y_{s}-\bar{\epsilon}$ giving a term proportional to $-\log \bar{\epsilon}$. The final result is then

$$
\lambda(s)=\delta(s)+\left[-\frac{\log \bar{\epsilon}}{2 \pi \rho} \lambda_{\text {cont }}(s)+\mathcal{O}\left(\bar{\epsilon}^{0}\right)\right] \Theta\left(s-m_{g}^{2}\right), \quad \lambda_{\text {cont }}(s)=\left(s-m_{g}^{2}\right)^{-1 / 2},
$$

where $\delta(s)$ is the contribution of the zero mode, $\lambda_{\text {cont }}(s)$ the contribution from the continuum, and the $\mathcal{O}\left(\bar{\epsilon}^{0}\right)$ term denotes the contribution from resonances. As we can see the contribution from the continuum is the dominant one and comes entirely from the singularity at $y_{s}$.

Finally, let us point out that the Green's function and spectral function can be written also in the form

$$
\begin{aligned}
& G_{A}\left(y, y^{\prime} ; s\right)=\frac{f_{0}(y) f_{0}\left(y^{\prime}\right)}{\left\|f_{0}\right\|^{2}} \frac{1}{s+i \epsilon}+\int_{m_{g}^{2}}^{\infty} d m^{2} \sigma\left(m^{2}\right) \frac{f_{m^{2}}(y) f_{m^{2}}\left(y^{\prime}\right)}{s-m^{2}+i \epsilon} \\
& \rho_{A}\left(y, y^{\prime} ; s\right)=\frac{f_{0}(y) f_{0}\left(y^{\prime}\right)}{\left\|f_{0}\right\|^{2}} \delta(s)+\sigma(s) f_{s}(y) f_{s}\left(y^{\prime}\right) \Theta\left(s-m_{g}^{2}\right)
\end{aligned}
$$

respectively, where $f_{0}(y)$ is the zero mode eigenfunction, $f_{m^{2}}(y)$ are continuum eigenfunctions, $\left\|f_{0}\right\|^{2} \equiv \int_{0}^{y_{s}} d y f_{0}(y)^{2}$ is the squared norm, and $\sigma(s)$ is a spectral density in the 

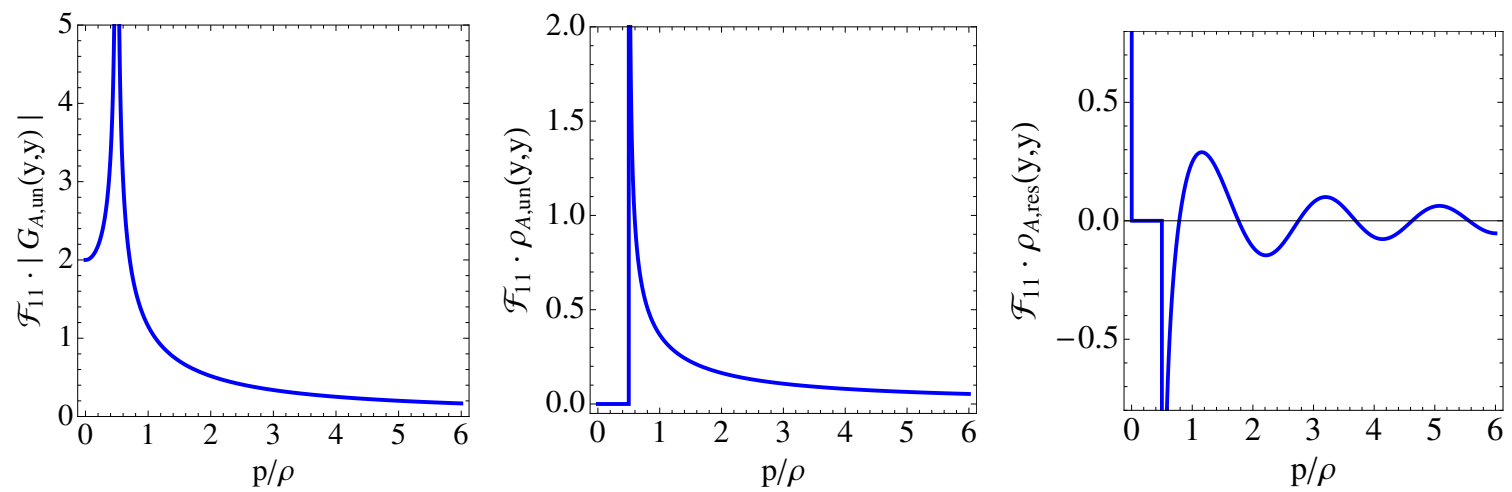

Figure 9. Plots of the unparticle and resonant contributions to the Green's function. We display the result for $\mathcal{F}_{11} \cdot\left|G_{A, \text { un }}\left(y, y^{\prime} ; p\right)\right|$ (left panel), $\mathcal{F}_{11} \cdot \rho_{A, \text { un }}\left(y, y^{\prime} ; p\right)$ (middle panel) and $\mathcal{F}_{11} \cdot \rho_{A, \text { res }}\left(y, y^{\prime} ; p\right)$ (right panel), as functions of $p / \rho$. We have used $y=y^{\prime}=\left(y_{1}+y_{s}\right) / 2$ and $A_{1}=35$ in all panels, and assume time-like momenta $p^{2}>0$.

continuum which is finite and whose relation with $\lambda(s)$ can be obtained by integrating eq. (3.64). These formulas generalize well-known expressions for the discrete case. A more detailed analysis of $\lambda(s)$ as well as the decomposition of eqs. (3.63)-(3.64) will be studied in a forthcoming publication [49].

\subsection{Unparticle vs. resonant contribution to Green's functions}

Once we have studied the resonances appearing in the Green's functions, related to the zeros of $\Phi(p)$, one question arises: can the Green's functions be considered just as summations of resonant contributions or, is there any genuine continuous contribution on top of the resonances? To answer this question, let us have a look at the explicit expression of the Green's function given by eq. (3.13). Notice that in the three regions $\left(y_{\downarrow}, y_{\uparrow} \leq y_{1}\right.$, $y_{\downarrow} \leq y_{1}<y_{\uparrow}$ and $\left.y_{1}<y_{\downarrow}, y_{\uparrow}\right)$, the function $1 / \Phi(p)$ multiplies the full expression. Taking into account the structure of the function $\mathcal{Q}(y)$ given by the last line of eq. (3.14), we can see that only in the last region one can split the Green's function into two terms

$$
G_{A}\left(y, y^{\prime} ; p\right)=G_{A, \mathrm{un}}\left(y, y^{\prime} ; p\right)+G_{A, \mathrm{res}}\left(y, y^{\prime} ; p\right), \quad y_{1}<y_{\downarrow}, y_{\uparrow},
$$

where

$$
\begin{aligned}
& G_{A, \text { un }}\left(y, y^{\prime} ; p\right)=-\frac{k}{\rho^{2}} \frac{1}{k\left(y_{s}-y_{\downarrow}\right)}\left(\frac{y_{s}-y_{\uparrow}}{y_{s}-y_{\downarrow}}\right)^{\Delta_{A}^{+} / 2} \frac{1}{\delta_{A}(p)}, \\
& G_{A, \text { res }}\left(y, y^{\prime} ; p\right)=\frac{k}{\rho^{2}}\left[k\left(y_{s}-y_{\downarrow}\right) \cdot k\left(y_{s}-y_{\uparrow}\right)\right]^{\Delta_{A}^{+} / 2} \frac{1}{\delta_{A}(p)} \cdot \frac{\Psi(p)}{\Phi(p)},
\end{aligned}
$$

corresponding to a continuous and a resonant contribution, respectively. The first contribution $G_{A \text {,un }} \propto \delta_{A}^{-1}$ is free of resonances, as $\Phi(p)$ does not appear in its expression, and it has a structure similar to the propagator of gapped unparticles [27]. We display in figure 9 the results for $\left|G_{A \text {, un }}(y, y ; p)\right|$, for the corresponding spectral function $\rho_{A \text {,un }}(y, y ; p)$, and for the resonant contribution to the spectral function $\rho_{A \text {,res }}(y, y ; p)$, with $y=\left(y_{1}+y_{s}\right) / 2$. Note 
that the unparticle contribution does not have any zero mode, and both the Green's function and the spectral function have divergent behaviors when the momentum approaches the mass gap from above, i.e. $\rho_{A, \text { un }}\left(y, y^{\prime}, p\right) \underset{p^{2} \rightarrow m_{g}^{2+}}{\longrightarrow}+\infty$.

An interesting property of the functions $\Phi(p)$ and $\Psi(p)$ is that ${ }^{14}$

$$
\Phi^{\mathrm{II}}(p)=\Psi^{\mathrm{I}}(p)
$$

where the superindexes I and II stand for the first and second Riemann sheet, respectively. This property implies that while $G_{A \text {,res }}\left(y, y^{\prime} ; p\right)$ has poles in the second Riemann sheet, it has zeros in the first Riemann sheet that are located in the complex $s$ plane at the same positions as the poles of the second Riemann sheet. Note, however, that these are not zeros of the full Green's function $G_{A}\left(y, y^{\prime} ; p\right)$, but only of the resonant part.

Finally notice that the (divergent) continuous eigenvalue of the $4 \mathrm{D}$ spectral function $\lambda(p)$, which was computed in section $3.4, \lambda_{\text {cont }}(s)$, corresponds to a contribution from unparticles with a dimension $d_{U}=3 / 2$ and mass gap $m_{g}[27,48]$.

\section{Gauge bosons with Dirichlet boundary condition}

In the considered extension of the SM we will use Dirichlet boundary condition for the extra gauge bosons $\left(W_{R}, Z_{R}\right)$ on the UV brane, see section 2.2. To study this, one should start from the general solution of eq. (3.7), which is given by eq. (3.8). While the Neumann boundary condition in the UV brane, used in eq. (3.10), leads to $\left(\partial_{y} G_{A}\right)\left(y_{0}\right)=0$, the Dirichlet boundary condition is given by

$$
G_{A}^{(-+)}\left(y_{0}\right)=0
$$

This condition is supplemented by the other conditions in eq. (3.10). As an example, the integration constants in Region I turn out to fulfill the relation

$$
C_{1}^{I}=-\frac{Y_{\alpha}\left(\frac{p}{k}\right)}{J_{\alpha}\left(\frac{p}{k}\right)} C_{2}^{I} \quad \text { with } \quad \alpha=\left\{\begin{array}{ll}
0 & (\text { Neumann }) \\
1 & \text { (Dirichlet) }
\end{array} .\right.
$$

As a consequence, the difference between the Green's functions with Neumann and Dirichlet boundary conditions will be in the indexes of some of the Bessel functions. Following a procedure similar to the one explained in section 3.1, the Green's functions with Dirichlet boundary condition turn out to be

$$
G_{A}^{(-+)}\left(y, y^{\prime} ; p\right)=\left\{\begin{array}{cc}
\frac{\pi}{2 k} e^{k\left(y+y^{\prime}\right) \frac{\overline{\mathcal{P}}\left(y_{\downarrow}\right) \mathcal{Z}\left(y_{\uparrow}\right)}{\Omega(p)}} & y_{\downarrow}, y_{\uparrow} \leq y_{1} \\
-\frac{2}{\rho} e^{k y_{\downarrow}}\left(k\left(y_{s}-y_{\uparrow}\right)\right)_{A}^{\Delta_{A}^{+} / 2} \frac{\overline{\mathcal{P}}\left(y_{\downarrow}\right)}{\Omega(p)} & y_{\downarrow} \leq y_{1}<y_{\uparrow} . \\
\left(\frac{y_{s}-y_{\uparrow}}{y_{s}-y_{\downarrow}}\right)^{\Delta_{A}^{+} / 2} \delta_{A}^{-1} \cdot \frac{\overline{\mathcal{Q}}\left(y_{\downarrow}\right)}{\Omega(p)} & y_{1}<y_{\downarrow}, y_{\uparrow}
\end{array}\right.
$$

\footnotetext{
${ }^{14}$ The property of eq. (3.68) follows from $\left(\Delta_{A}^{ \pm}\right)^{\mathrm{II}}=\left(\Delta_{A}^{\mp}\right)^{\mathrm{I}}$.
} 
We have used the notation

$$
\begin{aligned}
& \Omega(p)=\mathcal{Z}(0), \quad \bar{\Psi}(p)=Y_{1}(p / k) J_{-}(p / \rho)-J_{1}(p / k) Y_{-}(p / \rho), \\
& \overline{\mathcal{P}}(y)=Y_{1}(p / k) J_{1}\left(e^{k y} p / k\right)-J_{1}(p / k) Y_{1}\left(e^{k y} p / k\right), \\
& \overline{\mathcal{Q}}(y)=-\frac{k}{\rho^{2}} \frac{1}{k\left(y_{s}-y\right)}\left[\Omega(p)-\left(k\left(y_{s}-y\right)\right)^{\delta_{A}} \bar{\Psi}(p)\right]
\end{aligned}
$$

where the function $\mathcal{Z}(y)$ is defined in eq. (3.14). Note that in the limit $y \rightarrow y_{0}$ one has $\overline{\mathcal{P}}\left(y_{0}\right)=0$, so that

$$
G_{A}^{(-+)}\left(y_{0}, y^{\prime} ; p\right)=0
$$

a property that is consequence of the boundary condition of eq. (4.1). As in the case of gauge bosons with Neumann boundary condition in the UV brane, this Green's function also fulfills the property $G_{A}^{(-+)}\left(y, y^{\prime} ; p\right)=G_{A}^{(-+)}\left(y^{\prime}, y ; p\right)$.

The expression for the IR-to-IR Green's function $G_{A}^{(-+)}\left(y_{1}, y_{1} ; p\right)$ reduces to

$$
G_{A}^{(-+)}\left(y_{1}, y_{1} ; p\right)^{-1}=-\frac{\rho^{2}}{2 k} \cdot \frac{\Omega(p)}{\overline{\mathcal{P}}\left(y_{1}\right)} \simeq-\frac{\rho^{2}}{2 k}\left[\Delta_{A}^{+}+2 \frac{p}{\rho} \frac{J_{0}\left(\frac{p}{\rho}\right)}{J_{1}\left(\frac{p}{\rho}\right)}\right],
$$

where in the second equality we have assumed $p \ll k$, while the other brane-to-brane Green's functions, $G_{A}^{(-+)}\left(y_{0}, y_{0} ; p\right)$ and $G_{A}^{(-+)}\left(y_{0}, y_{1} ; p\right)$, are vanishing as a consequence of eq. (4.1), cf. eq. (4.5). The limit $p \ll \rho$ of the IR-to-IR Green's function is

$$
G_{A}^{(-+)}\left(y_{1}, y_{1} ; p\right)^{-1} \underset{p \ll \rho}{\simeq}-2 \frac{\rho^{2}}{k}+\frac{5}{4} \frac{p^{2}}{k}+\mathcal{O}\left(p^{4}\right),
$$

while its behaviors in the regime $\rho \ll p$ for time-like momenta, $p^{2}>0$, and space-like momenta, $p^{2}<0$, (and $p \ll k$ ) are the same as for the Green's function $G_{A}\left(y_{1}, y_{1} ; p\right)$, cf. eqs. (3.37) and (3.40).

In the following we denote the zero momentum limit of the IR-to-IR Green's function as $G_{A}^{(-+) 0}=-k /\left(2 \rho^{2}\right)$. We display in figure 10 the results for the normalized IR-to-IR Green's function $\left|G_{A}^{(-+)}\left(y_{1}, y_{1} ; p\right) / G_{A}^{(-+) 0}\right|$ for time-like momenta $p^{2}>0$ (left panel), and $G_{A}^{(-+)}\left(y_{1}, y_{1} ;|p|\right) / G_{A}^{(-+) 0}$ for space-like momenta $p^{2}<0$ (middle panel). In the latter case, the Green's function is purely real, and $G_{A}^{(-+)}\left(y_{1}, y_{1},|p|\right)$ decreases like the inverse power of $|p|$ for momenta $|p| \gg \rho$, i.e. $\sim \rho /|p|$.

Finally, it is displayed in the right panel of figure 10 the result for the rescaled spectral function $\mathcal{F}_{11} \cdot \rho_{A}^{(-+)}\left(y_{1}, y_{1} ; p\right)$, where the prefactor $\mathcal{F}_{11}$ is defined in eq. (3.31). Notice that gauge bosons with Dirichlet boundary conditions do not have zero modes, so that no Dirac delta behavior in the spectral function at $p=0$ is present in this case.

We now study the Green's function in the complex plane. One can see from eq. (4.3) that the pole structure of the Green's function $G_{A}^{(-+)}\left(y, y^{\prime} ; p\right)$ corresponds to the zeros of $\Omega(p)$. We display in the left panel of figure 11 a contour plot of $\log _{10}|\Omega(p)|$ in the second Riemann sheet, the lightest resonances appearing in the complex plane at

$$
(M / \rho, \Gamma / M)=(2.36,2.95),(5.98,1.13),(9.32,0.771),(12.59,0.603),(15.82,0.501), \cdots .
$$



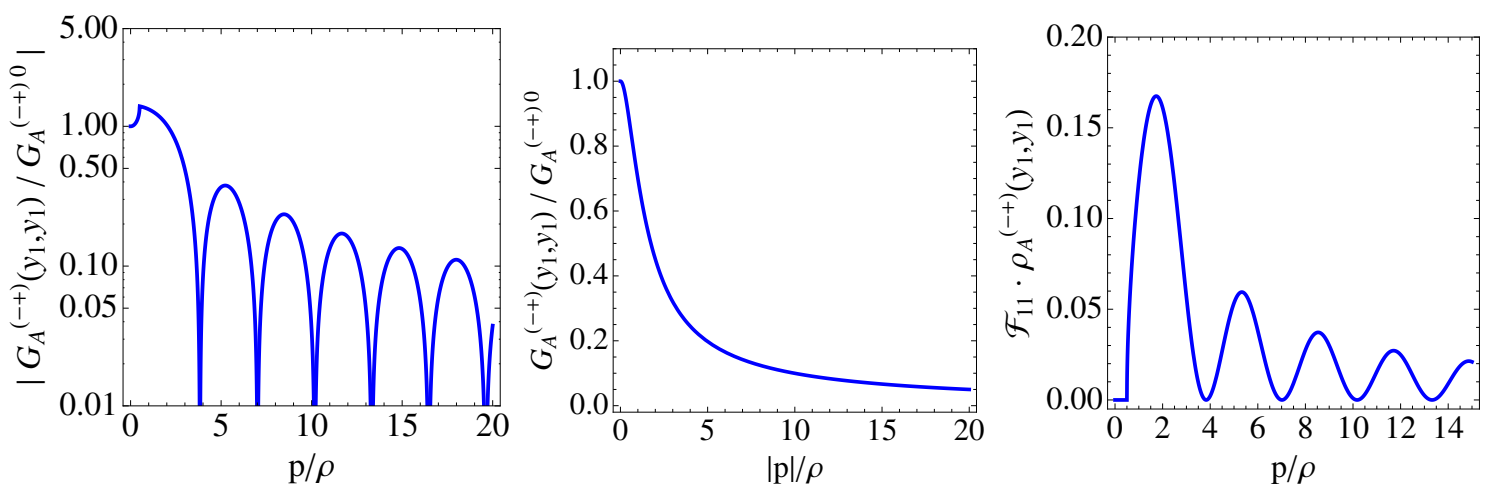

Figure 10. Plots of the IR-to-IR Green's function $\left|G_{A}^{(-+)}\left(y_{1}, y_{1} ; p\right) / G_{A}^{(-+) 0}\right|$ for $p^{2}>0$ (left panel), $G_{A}^{(-+)}\left(y_{1}, y_{1} ;|p|\right) / G_{A}^{(-+) 0}$ for $p^{2}<0$ (middle panel), and the rescaled spectral function $\mathcal{F}_{11} \cdot \rho_{A}^{(-+)}\left(y_{1}, y_{1} ; p\right)$ (right panel) as functions of $p / \rho$. We have used $A_{1}=35$ in all panels.
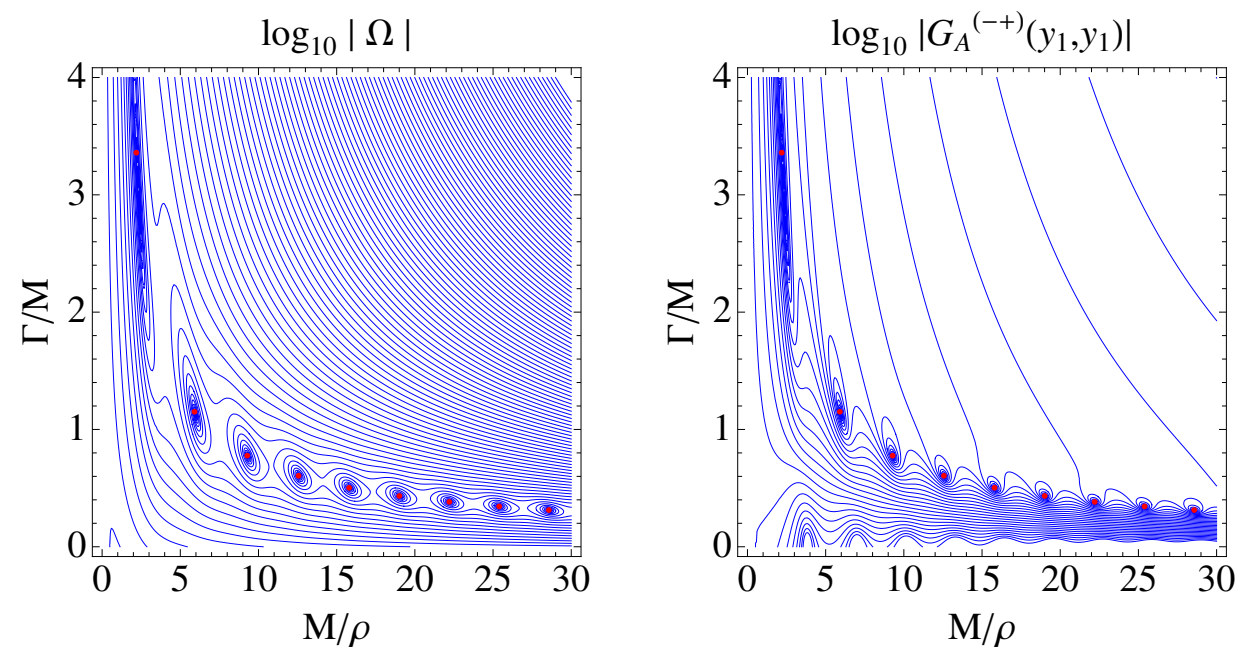

Figure 11. Contour plot in the plane $(M / \rho, \Gamma / M)$ of $\log _{10}|\Omega(p)|$ cf. eq. (3.14) (left panel), and the absolute value of the IR-to-IR Green's function $\log _{10}\left|G_{A}^{(-+)}\left(y_{1}, y_{1}\right)\right|$ (right panel). The (red) dots stand for the positions of the poles of the Green's function with Dirichlet boundary condition as predicted by eq. (4.9), and given by the analytical formula of eq. (3.46). We have considered $A_{1}=35$.

We can analytically study these zeros in a way similar to the procedure explained in section 3.3. The expansion of $\Omega(p)$ at large momentum $\rho \ll|p|(|p| \ll k)$ leads to

$$
\Omega(p) \underset{\rho \ll|p|}{\simeq} \frac{e^{-i(p / \rho+\pi / 4)}}{\sqrt{2 \pi^{3}}}\left[e^{i 2 p / \rho}-8 i\left(\frac{p}{\rho}\right)^{2}\right] \frac{k}{\rho}\left(\frac{\rho}{p}\right)^{5 / 2}, \quad \operatorname{Im}\left((p / \rho)^{2}\right)<0 .
$$

This asymptotic behavior is similar to the one of $\Phi(p)$, cf. eq. (3.44), hence we find that at this order of the computation the zeros of $\Omega(p)$ are located at the same positions as the zeros of $\Phi(p)$, and they are given by eq. (3.46). We conclude that the poles of the Green's function for gauge bosons $G_{A}^{(-+)}\left(y, y^{\prime} ; p\right)$ are located very close to the poles of $G_{A}\left(y, y^{\prime} ; p\right)$. The relative difference between the location of the poles in both cases is $\lesssim 2 \%$ for the lightest resonances, and $\lesssim 0.5 \%$ for resonances $M / \rho \gtrsim 10$. Finally, let us mention that 
the relative error of the approximate formula of eq. (3.46) with respect to the true zeros of $\Omega(p)$ is a factor $2-3$ better than for the case of $\Phi(p)$, except for the lightest resonance, for which is similar, cf. section 3.3.

The Green's function for gauge bosons with Dirichlet boundary condition in the UV brane also has unparticle and resonant contributions, as for the massless case in eq. (3.65). The unparticle contribution turns out to be identical as for massless gauge bosons, and it is given by eq. (3.66).

Finally, we can study as well the positivity of the spectral operator $\hat{\rho}_{A}^{(-+)}$. The procedure is similar to the one presented in section 3.4, leading to a single non-vanishing eigenvalue $\lambda^{(-+)}(p)$ given by the trace of the matrix $\left(\hat{\rho}_{A}^{(-+)}\right)_{y}^{y^{\prime}}$. As for the case of gauge bosons with Neumann boundary conditions, the integral over the $y$ coordinate has to be regularized with a cutoff $\bar{\epsilon}$, leading to a divergent unparticle contribution from the region $y_{1}<y<y_{s}$, and other finite contribution from the resonances. The result is given by eq. (3.62) but without the pole at the origin, i.e.

$$
\lambda^{(-+)}(s)=\left[-\frac{\log \bar{\epsilon}}{2 \pi \rho} \lambda_{\text {un }}(s)+\mathcal{O}\left(\bar{\epsilon}^{0}\right)\right] \Theta\left(s-m_{g}^{2}\right), \quad \lambda_{\text {un }}(s)=\left(s-m_{g}^{2}\right)^{-1 / 2},
$$

where $\lambda_{\text {un }}(s)$ is the spectral function of an unparticle with a mass gap $m_{g}$ and dimension $d_{U}=3 / 2$.

\section{$5 \quad$ Electroweak precision observables}

Even if the observable $T$ is protected by the custodial symmetry, as it is well known the rest of observables are unprotected and, as our model departs from the usual RS models since resonances have a (broad) width, it is worth doing a detailed analysis of (oblique) electroweak observables.

When the electroweak symmetry is broken there is a mixing between the SM fields $W_{L}$ and $Z_{L}$ and the heavy modes of $W_{L, R}$ and $Z_{L, R}$ induced by the Lagrangian

$$
\mathcal{L}=\operatorname{tr}\left|g_{L}^{5} W_{L}^{a} T_{L}^{a} \mathcal{H}-g_{R}^{5} \mathcal{H} W_{R}^{a} T_{R}^{a}\right|^{2},
$$

where we are indicating with the script $g_{5}$ the $5 \mathrm{D}$ gauge couplings, related to the $4 \mathrm{D}$ couplings $g_{4}$ by $g_{5}=g_{4} \sqrt{y_{s}}$.

After putting the Higgs bi-doublet $\mathcal{H}$, which we assume to be localized on the IR brane,

$$
\mathcal{H}=\left(\begin{array}{cc}
H_{2}^{0} & H_{1}^{-} \\
H_{2}^{-} & H_{1}^{0}
\end{array}\right)
$$

at its minimum, $\left\langle H_{1,2}^{0}\right\rangle=v_{1,2}$, with $v_{1}^{2}+v_{2}^{2}=v^{2}$ and $v=246.22 \mathrm{GeV}$, the Lagrangian (5.1) gives rise to the quadratic terms

$$
\begin{aligned}
\mathcal{L}=\frac{v^{2}}{4} y_{s} & {\left[g_{L}^{2} W_{L}\left(y_{1}, x\right) W_{L}\left(y_{1}, x\right)+g_{R}^{2} W_{R}\left(y_{1}, x\right) W_{R}\left(y_{1}, x\right)-\frac{2 v_{1} v_{2}}{v^{2}} g_{L} g_{R} W_{L}\left(y_{1}, x\right) W_{R}\left(y_{1}, x\right)\right.} \\
+ & \left.\frac{1}{2} \frac{g_{L}^{2}}{c_{L}^{2}} Z_{L}\left(y_{1}, x\right) Z_{L}\left(y_{1}, x\right)+\frac{1}{2} g_{R}^{2} c_{R}^{2} Z_{R}\left(y_{1}, x\right) Z_{R}\left(y_{1}, x\right)-g_{L} g_{R} \frac{c_{R}}{c_{L}} Z_{L}\left(y_{1}, x\right) Z_{R}\left(y_{1}, x\right)\right],
\end{aligned}
$$


where $W_{X} W_{X} \equiv W_{X}^{-} W_{X}^{+}$for $X=L, R$, and $W_{L} W_{R} \equiv W_{L}^{-} W_{R}^{+}+W_{L}^{+} W_{R}^{-}$. One has $v_{1}=v \cdot \cos \beta$ and $v_{2}=v \cdot \sin \beta$, and then $2 v_{1} v_{2} / v^{2}=2 t_{\beta} /\left(1+t_{\beta}^{2}\right)$ where we have defined $t_{\beta} \equiv \tan \beta$. In the custodial limit $t_{\beta}=1$ and $v_{1}=v_{2}=v / \sqrt{2}$.

As we have seen in the previous sections and in the appendix $\mathrm{A}$, the fields $W_{L}$ and $Z_{L}$ have a zero mode, which is the corresponding SM field, and a gapped continuum of states, while the fields $W_{R}$ and $Z_{R}$ do not possess zero mode, but only the continuum of states above the mass gap. For the electroweak observables contributing to the new physics, the oblique $T, S$ and $U$ parameters are defined as [50]

$$
\begin{aligned}
\alpha T & =\frac{\Pi_{W W}(0)}{m_{W}^{2}}-\frac{\Pi_{Z Z}(0)}{m_{Z}^{2}}, \\
\alpha S & =4 s_{L}^{2} c_{L}^{2} \Pi_{Z Z}^{\prime}(0), \\
\alpha(S+U) & =4 s_{L}^{2} \Pi_{W W}^{\prime}(0) .
\end{aligned}
$$

For the computation of these parameters, we need to select as external fields the zero modes of either $W_{L}$ and $Z_{L}$ and only propagate the continuum of states. We will then define the Green's functions propagating only the continuum of states as

$$
\begin{aligned}
& \mathcal{G}_{W_{L}, Z_{L}}\left(y_{1}, y_{1} ; p\right)=G_{W_{L}, Z_{L}}\left(y_{1}, y_{1} ; p\right)-G_{W_{L}, Z_{L}}^{0}(p), \\
& \mathcal{G}_{W_{R}, Z_{R}}\left(y_{1}, y_{1} ; p\right)=G_{W_{R}, Z_{R}}\left(y_{1}, y_{1} ; p\right)
\end{aligned}
$$

where $G_{W_{L}, Z_{L}}$ and $G_{W_{R}, Z_{R}}$ are, respectively, the Green's functions $G_{A}$ and $G_{A}^{(-+)}$computed in sections 3 and $4 .{ }^{15}$ By using the notation $\mathcal{G}(0) \equiv \lim _{p \rightarrow 0} \mathcal{G}\left(y_{1}, y_{1} ; p\right)$, a straightforward calculation yields [44]

$$
\alpha T=m_{W}^{2} y_{s}\left[\mathcal{G}_{W_{L}}(0)+\frac{4 t_{\beta}^{2}}{\left(1+t_{\beta}^{2}\right)^{2}} \frac{g_{R}^{2}}{g_{L}^{2}} \mathcal{G}_{W_{R}}(0)\right]-m_{Z}^{2} y_{s}\left[\mathcal{G}_{Z_{L}}(0)+\frac{g_{R}^{2}}{g_{L}^{2}} c_{L}^{2} c_{R}^{2} \mathcal{G}_{Z_{R}}(0)\right],
$$

where $t_{\beta}=v_{2} / v_{1}$. Using the results of previous sections we find

$$
\begin{aligned}
& \mathcal{G}_{W_{L}}(0)=\mathcal{G}_{Z_{L}}(0)=\frac{-2\left(k y_{s}\right)^{2}+6\left(k y_{s}\right)-9}{4\left(k y_{s}\right) y_{s} \rho^{2}} \equiv \mathcal{G}_{L}(0), \\
& \mathcal{G}_{W_{R}}(0)=\mathcal{G}_{Z_{R}}(0)=-\frac{\left(k y_{s}\right)}{2 y_{s} \rho^{2}} \equiv \mathcal{G}_{R}(0),
\end{aligned}
$$

so that

$$
\alpha T=m_{W}^{2} y_{s} \frac{s_{L}^{2}}{c_{L}^{2}}\left[\left(1-\frac{1}{s_{R}^{2}} \frac{\left(1-t_{\beta}^{2}\right)^{2}}{\left(1+t_{\beta}^{2}\right)^{2}}\right) \mathcal{G}_{R}(0)-\mathcal{G}_{L}(0)\right] .
$$

A similar calculation yields

$$
\begin{aligned}
& \alpha S=4 m_{Z}^{4} y_{s} s_{L}^{2} c_{L}^{2}\left[\mathcal{G}_{L}^{\prime}(0)+\frac{s_{L}^{2} c_{R}^{2}}{s_{R}^{2}} \mathcal{G}_{R}^{\prime}(0)\right] \\
& \alpha U=4 m_{Z}^{4} y_{s} s_{L}^{4} c_{L}^{2}\left[\left(1-\frac{1}{s_{R}^{2}} \frac{\left(1-t_{\beta}^{2}\right)^{2}}{\left(1+t_{\beta}^{2}\right)^{2}}\right) \mathcal{G}_{R}^{\prime}(0)-\mathcal{G}_{L}^{\prime}(0)\right]
\end{aligned}
$$

\footnotetext{
${ }^{15}$ In this section we are neglecting the finite mass effects of $W$ and $Z$ bosons, an approximation which is valid as long as $m_{W, Z} \ll \rho$. These effects could have been easily considered by using the propagators of appendix A and evaluating the expressions of eq. (5.4) at the pole of the Green's function $G_{A, M}\left(y_{1}, y_{1} ; p^{2}\right)$, i.e. at $p^{2} \simeq m_{W, Z}^{2}$. However these effects should be negligible in view of the mass hierarchy $m_{A} \ll \rho$.
} 

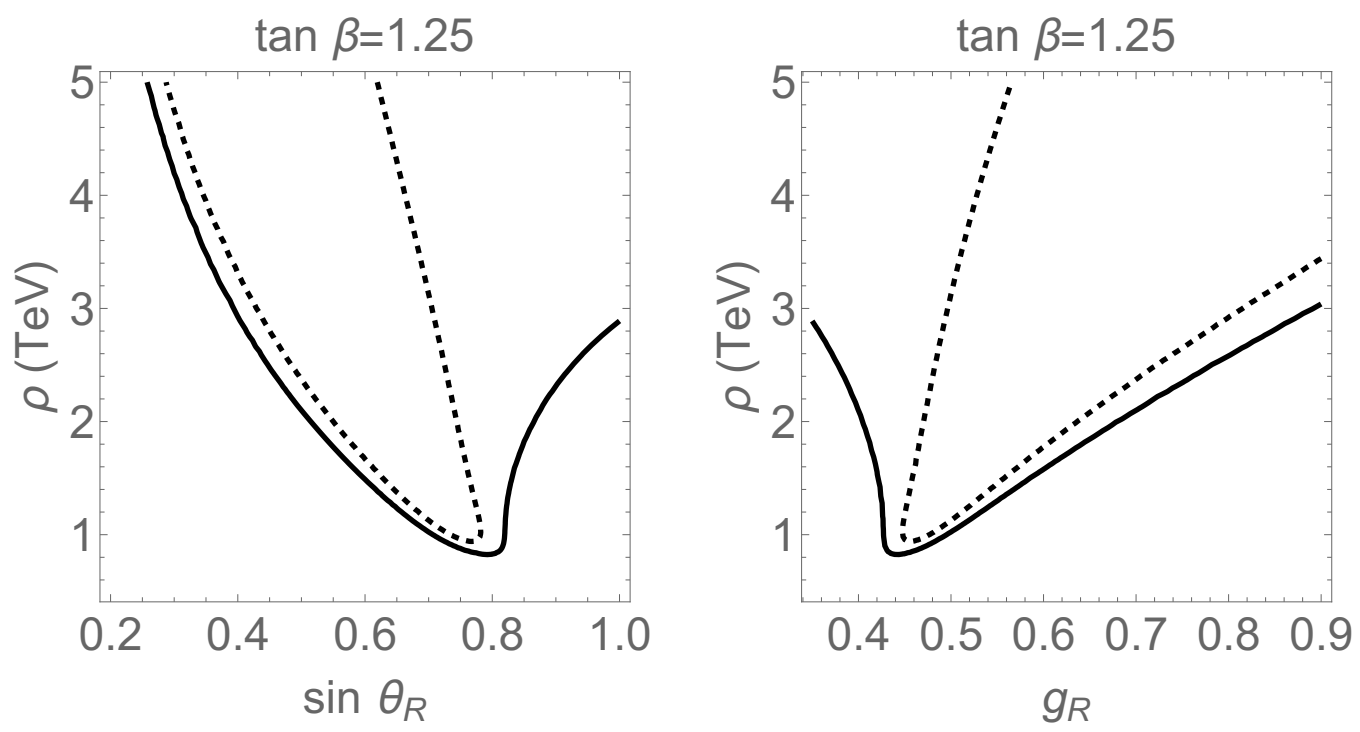

Figure 12. $\chi^{2}$ distribution in the plane $\left(s_{R}, \rho\right)$ (left panel) and $\left(g_{R}, \rho\right)$ (right panel) for the value of $t_{\beta}=1.25$. The solid (dashed) lines are the corresponding $95 \%$ (67\%) C.L. limits.

where the prime stands for $\frac{d}{d p^{2}}$, and $\mathcal{G}_{W_{L}}^{\prime}(0)=\mathcal{G}_{Z_{L}}^{\prime}(0) \equiv \mathcal{G}_{L}^{\prime}(0), \mathcal{G}_{W_{R}}^{\prime}(0)=\mathcal{G}_{Z_{R}}^{\prime}(0) \equiv \mathcal{G}_{R}^{\prime}(0)$. Then we find

$$
\begin{aligned}
\mathcal{G}_{L}^{\prime}(0) & =\frac{-40\left(k y_{s}\right)^{3}+324\left(k y_{s}\right)^{2}-977\left(k y_{s}\right)+648}{128\left(k y_{s}\right)^{2} y_{s} \rho^{4}}, \\
\mathcal{G}_{R}^{\prime}(0) & =-\frac{5\left(k y_{s}\right)}{16 y_{s} \rho^{4}} .
\end{aligned}
$$

We can see that, in the limit of large value of $k y_{s}, \mathcal{G}_{L, R}(0), \mathcal{G}_{L, R}^{\prime}(0)=\mathcal{O}\left(k y_{s}\right)$, while $\mathcal{G}_{R}(0)-\mathcal{G}_{L}(0), \mathcal{G}_{R}^{\prime}(0)-\mathcal{G}_{L}^{\prime}(0)=\mathcal{O}\left(k y_{s}\right)^{0}$, which is the cancellation which appears on the observables $T$ and $U$ in the custodial limit $t_{\beta}=1$. However, still the observable $S$ gives a sizable contribution, which is partly cancelled if we introduce a small breaking of the custodial symmetry, i.e. when we introduce a small value of $t_{\beta}-1$.

The most recent experimental constraints for the oblique $S, T$ and $U$ parameters [51] gives

$$
S=-0.01 \pm 0.10, \quad T=0.03 \pm 0.12, \quad U=0.02 \pm 0.11
$$

with correlations

$$
\operatorname{corr}(S, T)=92 \%, \quad \operatorname{corr}(S, U)=-80 \%, \quad \operatorname{corr}(T, U)=-93 \% .
$$

We display in figure 12 the $\chi^{2}$ distribution in the plane $\left(s_{R}, \rho\right)$ (left panel) and $\left(g_{R}, \rho\right)$ (right panel) for the value of $t_{\beta}=1.25$. The solid (dashed) lines are the corresponding 95\% (67\%) C.L. limits. As we can see the lowest value of the parameter $\rho$ is $\rho_{\min } \sim 1 \mathrm{TeV}$, which corresponds to a value of the coupling $g_{R} \sim 0.45$, in turn corresponding to $s_{R} \sim 0.8$, well in the perturbative region. For other values of $t_{\beta}$ the result smoothly changes. For instance in the custodial limit, i.e. for $t_{\beta}=1$ we find that $\rho_{\min } \sim 2.5 \mathrm{TeV}$, corresponding to a value of $g_{R} \sim 5.6$, well inside the non-perturbative region. 


\section{Conclusions and outlook}

In this paper we have studied a 5D model which naturally leads to gapped continuum spectra. The model is defined in terms of a metric which has an $\mathrm{AdS}_{5}$ behavior in the UV, and a linear behavior in the IR for the scalar field in conformal coordinates, and constitutes a faithful enough approximation of the model presented in ref. [13], having the advantage of allowing for analytical expressions for the Green's functions. In this paper we have concentrated on the case of bulk propagating gauge bosons $A_{M}$, with Green's functions $G_{A}\left(y, y^{\prime}\right)$. The spectrum of SM massless gauge bosons (the photon and gluon) is a continuum of $\mathrm{KK}$ modes with a mass gap equal to $m_{g}=\rho / 2$ where $\rho \sim \mathrm{TeV}$, and an isolated massless pole which corresponds to the corresponding 4D gauge boson. In the case of SM massive gauge bosons (the $W$ and $Z$ bosons) the isolated pole becomes massive. For the case of gauge bosons with Dirichlet boundary conditions on the UV brane, the spectrum is a continuum with a mass gap equal to $m_{g}$, but without any isolated pole, which has been projected out of the spectrum by the boundary conditions.

We have considered the Green's functions in the complex $s$ plane, and found the existence of poles in the second Riemann sheet. We have computed the masses and widths of the associated resonances, and found that, while the former are close to the masses of the KK modes in the RS model, the latter are quite large $(\Gamma / \rho \gtrsim 7)$ indicating the presence of broad resonances. Their relative widths $\Gamma / M$, however, decrease with increasing energy, so that they tend to a distribution closer to Dirac delta functions in this regime. The behavior of the Green's functions $G_{A}\left(y, y^{\prime}\right)$ with $y_{1}<y, y^{\prime}$, i.e. between the IR brane and the singularity, is then explained as a summation of two contributions: a contribution which is purely continuous, and turns out to be related to gapped unparticle propagators, and other contribution that contains the resonances. We have extended theses analyses to gauge bosons with Dirichlet boundary condition in the UV brane, as well as to massive gauge bosons, leading to similar conclusions. In all the cases the resonances are present: in the Dirichlet case the positions of the poles in the complex plane are very close to the poles for massless gauge bosons, while in the massive case the values of the widths turn out to decrease with increasing values of $m_{A} / \rho$.

Notice that while the RS model leads to a discrete KK spectrum with zero widths [3], the linear dilaton model has a purely continuous spectrum above the mass gap, apart from the possible existence of isolated zero modes [15] (see also [14]). As our model shares both features, a RS metric between the UV and IR branes, and a linear dilaton model metric between the IR brane and the singularity, the result is that the RS resonances are endowed with a width, as a result of the effect of the linear dilaton metric while there still remains a pure unparticle contribution to the Green's functions. In fact our explicit Green's functions exhibit a pole structure in the second Riemann of the complex $s$ plane, with a broad width, which widely depart from an infinite series of Breit-Wigner resonances. Notice that in spite of being a 4D Green's function the particle width is incorporated $a b$ initio and is not associated to particle production, a characteristic feature of unparticles. We plan to go deeper into this issue in the future. 
This present study can be extended to the computation of Green's functions of other fields, i.e. fermions, Higgs bosons, the graviton and the radion. Regarding phenomenological applications, the brane-to-brane Green's functions can be used to study the excess with respect to the SM prediction of some processes at the LHC, in particular the cross-section of $p p$ collisions where a continuum KK gluon is produced by Drell-Yan processes and decays into a pair of light/heavy fermions localized in the UV/IR brane. For other phenomenological applications in particle physics, it would be interesting to study the couplings of the continuum KK modes with the SM fields, and provide values for the Wilson coefficients of the corresponding effective field theory. Other possible applications include the study of dark matter as a weakly interacting continuum [16, 52]. Some of these issues will be addressed in a forthcoming publication [49].

\section{Acknowledgments}

We would like to thank A. Carmona, M. Pérez-Victoria and L.L. Salcedo for fruitful discussions. The authors thank the ICTP South American Institute for Fundamental Research (SAIFR), Sao Paulo, Brazil, and its Program on Particle Physics, September 30-November 30, 2019, where part of this work was done, for hospitality. The work of EM is supported by the Spanish MINEICO under Grants FIS2017-85053-C2-1-P and PID2020-114767GB-I00, by the FEDER/Junta de Andalucía-Consejería de Economía y Conocimiento 2014-2020 Operational Programme under Grant A-FQM-178-UGR18, by Junta de Andalucía under Grant FQM-225, and by the Consejería de Conocimiento, Investigación y Universidad of the Junta de Andalucía and European Regional Development Fund (ERDF) under Grant SOMM17/6105/UGR. The research of EM is also supported by the Ramón y Cajal Program of the Spanish MINEICO under Grant RYC-2016-20678. The work of MQ is partly supported by Spanish MINEICO under Grant FPA2017-88915-P, by the Catalan Government under Grant 2017SGR1069, and by Severo Ochoa Excellence Program of MINEICO under Grant SEV-2016-0588. IFAE is partially funded by the CERCA program of the Generalitat de Catalunya.

\section{A Standard model massive gauge bosons}

In this appendix we will study the Green's functions $G_{A, M}$ for massive SM gauge bosons. As we are considering the Higgs sector localized on the IR brane, in the case of the SM massive gauge bosons $A_{\mu}$ there are extra terms in the 5D Lagrangian, eq. (3.1), as

$$
\Delta \mathcal{L}_{5}=\left(-\frac{1}{2} M_{Z}^{2} Z_{\mu}^{2}-M_{W}^{2}\left|W_{\mu}\right|^{2}\right) \delta\left(y-y_{1}\right), \quad M_{A}^{2}=y_{s} m_{A}^{2},
$$

(for $A=W, Z$ ), which leads to a modification of the EoM for the Green's function, as

$$
\left[p^{2}-y_{s} m_{A}^{2} \delta\left(y-y_{1}\right)\right] G_{A, M}\left(y, y^{\prime} ; p\right)+\partial_{y}\left(e^{-2 A} \partial_{y} G_{A, M}\left(y, y^{\prime} ; p\right)\right)=\delta\left(y-y^{\prime}\right)
$$


Using eq. (A.2), the derivative of the Green's functions turns out to be discontinuous at $y=y_{1}$, with a jump given by ${ }^{16}$

$$
\left.\Delta\left(\partial_{y} G_{A, M}\right)\left(y, y^{\prime}\right)\right|_{y=y_{1}}=m_{A}^{2} y_{s} e^{2 A\left(y_{1}\right)} G_{A, M}\left(y_{1}, y^{\prime}\right) .
$$

One can now solve the EoM by dividing the $y$ space into three regions, as explained in section 3.1. When doing that, we find a general solution identical to eq. (3.8) which is subject to the same boundary and matching conditions as in eq. (3.10), except for $\Delta\left(\partial_{y} G_{A, M}\right)\left(y_{1}\right)$ which is given by eq. (A.3). Finally, one finds that the Green's function for massive gauge bosons is given by eq. (3.13) with the replacements $\Phi(p) \rightarrow \Phi_{M}(p)$, $\mathcal{Z}(y) \rightarrow \mathcal{Z}_{M}(y)$ and $\mathcal{Q}(y) \rightarrow \mathcal{Q}_{M}(y)$, where the functions $\Phi_{M}(p), \mathcal{Z}_{M}(y)$ and $\mathcal{Q}_{M}(y)$ are given by

$$
\begin{aligned}
& \Phi_{M}(p)=Y_{0}(p / k) \cdot J_{M+}(p / \rho)-J_{0}(p / k) \cdot Y_{M+}(p / \rho), \\
& \Psi_{M}(p)=Y_{0}(p / k) \cdot J_{M-}(p / \rho)-J_{0}(p / k) \cdot Y_{M-}(p / \rho), \\
& \mathcal{Z}_{M}(y)=J_{M+}(p / \rho) \cdot Y_{1}\left(e^{k y} p / k\right)-Y_{M+}(p / \rho) \cdot J_{1}\left(e^{k y} p / k\right), \\
& \mathcal{Q}_{M}(y)=-\frac{k}{\rho^{2}} \frac{1}{k\left(y_{s}-y\right)}\left[\Phi_{M}(p)-\left(k\left(y_{s}-y\right)\right)^{\delta_{A}} \Psi_{M}(p)\right],
\end{aligned}
$$

with

$$
J_{M \pm}(p / \rho)=2 \frac{p}{\rho} J_{0}(p / \rho)+\Xi_{A}^{ \pm} J_{1}(p / \rho), \quad Y_{M \pm}(p / \rho)=2 \frac{p}{\rho} Y_{0}(p / \rho)+\Xi_{A}^{ \pm} Y_{1}(p / \rho),
$$

and we have used the notation

$$
\Xi_{A}^{ \pm}=\Delta_{A}^{ \pm}+2 k y_{s} \cdot\left(m_{A} / \rho\right)^{2}
$$

The approximate expressions of $\Phi_{M}(p)$ and $\Psi_{M}(p)$ for $p \ll k$ turn out to be

$$
\Phi_{M}(p)=\mathcal{K} J_{M+}(p / \rho)-Y_{M+}(p / \rho), \quad \Psi_{M}(p)=\mathcal{K} J_{M-}(p / \rho)-Y_{M-}(p / \rho) .
$$

In the limit $y \rightarrow y_{0}$ one finds the same expression as eq. (3.18) with the replacements for $\Phi$ and $\mathcal{Z}$ mentioned above. As it is obvious from eq. (A.6), the present Green's function tends to the result for massless gauge bosons given by eq. (3.13), when considering the limit $m_{A} \rightarrow 0\left(\Xi_{A}^{ \pm} \rightarrow \Delta_{A}^{ \pm}\right)$. As in the massless case, the Green's function for massive gauge bosons fulfills the property $G_{A, M}\left(y, y^{\prime} ; p\right)=G_{A, M}\left(y^{\prime}, y ; p\right)$.

\footnotetext{
${ }^{16}$ In the following we will assume that $y^{\prime} \neq y_{1}$, hence $\int_{y_{1}-\epsilon}^{y_{1}+\epsilon} d y \delta\left(y-y^{\prime}\right)=0$ and the term in the righthand side of eq. (A.2) does not contribute to eq. (A.3). Then, the Green's functions involving the IR brane are computed as $G_{A, M}\left(y, y_{1}\right)=\lim _{y^{\prime} \rightarrow y_{1}} G_{A, M}\left(y, y^{\prime}\right)$. Alternatively we could directly compute the Green's function $G_{A, M}\left(y, y_{1}\right)$ by considering a jump at $y=y_{1}$ given by

$$
\left.\Delta\left(\partial_{y} G_{A, M}\right)\left(y, y_{1}\right)\right|_{y=y_{1}}=m_{A}^{2} y_{s} e^{2 A\left(y_{1}\right)} G_{A, M}\left(y_{1}, y_{1}\right)+e^{2 A\left(y_{1}\right)},
$$

where the first and second term in the right-hand side correspond to the contribution of the terms $\propto \delta\left(y-y_{1}\right)$ and $\propto \delta\left(y-y^{\prime}\right)$ in eq. (A.2), respectively. We have checked that both procedures lead to the same result.
} 
The analytical expressions for the brane-to-brane Green's functions are, respectively,

$$
\begin{aligned}
& G_{A, M}^{-1}\left(y_{0}, y_{0} ; p\right)=\frac{p \Phi_{M}(p)}{\mathcal{Z}_{M}\left(y_{0}\right)} \simeq-\frac{\pi p^{2}}{2 k} \cdot \frac{\Phi_{M}(p)}{J_{M+}(p)} \\
& G_{A, M}^{-1}\left(y_{0}, y_{1} ; p\right)=-\frac{\pi}{4} \frac{\rho}{k} p \Phi_{M}(p) \\
& G_{A, M}^{-1}\left(y_{1}, y_{1} ; p\right)=-\frac{\rho^{2}}{2 k} \cdot \frac{\Phi_{M}(p)}{\mathcal{P}\left(y_{1}\right)} \simeq-\frac{\rho^{2}}{2 k} \cdot \frac{\Phi_{M}(p)}{\mathcal{K} \cdot J_{1}\left(\frac{p}{\rho}\right)-Y_{1}\left(\frac{p}{\rho}\right)}
\end{aligned}
$$

where in the second equality of eqs. (A.8) and (A.10) we have assumed $p \ll k$, and in the limit $p, m_{A} \ll \rho$ they are

$$
\begin{aligned}
G_{A, M}^{-1}\left(y_{0}, y_{0} ; p\right) \simeq & -\left[\frac{1}{k y_{s}\left(m_{A} / \rho\right)^{2}}+\frac{1}{2}\right]^{-1} \frac{\rho^{2}}{k} \\
& +\left[1-\frac{3}{2}\left(m_{A} / \rho\right)^{2}\right] y_{s} p^{2}+\mathcal{O}\left(\left(m_{A} / \rho\right)^{4},(p / \rho)^{4}\right) \\
G_{A, M}^{-1}\left(y_{0}, y_{1} ; p\right) \simeq & -y_{s} m_{A}^{2} \\
& +\left[1+\frac{1}{2}\left(-\frac{3}{2}+k y_{s}\right)\left(m_{A} / \rho\right)^{2}\right] y_{s} p^{2}+\mathcal{O}\left(\left(m_{A} / \rho\right)^{4},(p / \rho)^{4}\right) \\
G_{A, M}^{-1}\left(y_{1}, y_{1} ; p\right) \simeq & -y_{s} m_{A}^{2}+y_{s} p^{2}+\mathcal{O}\left(\left(m_{A} / \rho\right)^{4},(p / \rho)^{4}\right)
\end{aligned}
$$

These functions have poles at

$$
\begin{aligned}
& \left.p^{2}\right|_{\left(y_{0}, y_{0}\right)} \simeq m_{A}^{2}\left[1+\left(3-k y_{s}\right) \frac{m_{A}^{2}}{2 \rho^{2}}+\mathcal{O}\left(\left(m_{A} / \rho\right)^{4}\right)\right], \\
& \left.p^{2}\right|_{\left(y_{0}, y_{1}\right)} \simeq m_{A}^{2}\left[1+\left(\frac{3}{2}-k y_{s}\right) \frac{m_{A}^{2}}{2 \rho^{2}}+\mathcal{O}\left(\left(m_{A} / \rho\right)^{4}\right)\right], \\
& \left.p^{2}\right|_{\left(y_{1}, y_{1}\right)} \simeq m_{A}^{2}\left[1+\mathcal{O}\left(\left(m_{A} / \rho\right)^{4}\right)\right] .
\end{aligned}
$$

We show in figure 13 the inverse Green's functions $G_{A, M}^{-1}\left(y_{0}, y_{0} ; p\right), G_{A, M}^{-1}\left(y_{0}, y_{1} ; p\right)$ and $G_{A, M}^{-1}\left(y_{1}, y_{1} ; p\right)$ as functions of $p$, for $\rho=1 \mathrm{GeV}$ (left panel), $\rho=4 \mathrm{GeV}$ (right panel) and $m_{A}=m_{Z}$. The position of the zero in these panels corresponds to the pole in $G_{A}\left(y_{\alpha}, y_{\alpha} ; p\right)$. Notice that the Green's functions are real in the range of momenta $0 \leq p<m_{g}$ (except for a Dirac delta behavior at $p \simeq m_{A}$ as we will see below). We have normalized the plots by the values of the Green's functions at $p=0$, i.e.

$$
G_{A, M}^{0}=\lim _{p \rightarrow 0} G_{A, M}\left(y, y^{\prime} ; p\right) \simeq-\frac{1}{y_{s} m_{A}^{2}} .
$$

The asymptotic behaviors for the inverse Green's functions with time-like momenta $p^{2}>0$, $p \gg \rho$, and with space-like momenta $p^{2}<0,|p| \gg \rho$, are given, respectively, by eqs. (3.35)(3.37) and (3.38)-(3.40).

In order to study the poles in the (second Riemann sheet of the) complex $s$ plane of the Green's functions, we display in figure 14 a contour plot of $\log _{10}\left|\Phi_{M}(p)\right|$, where the 

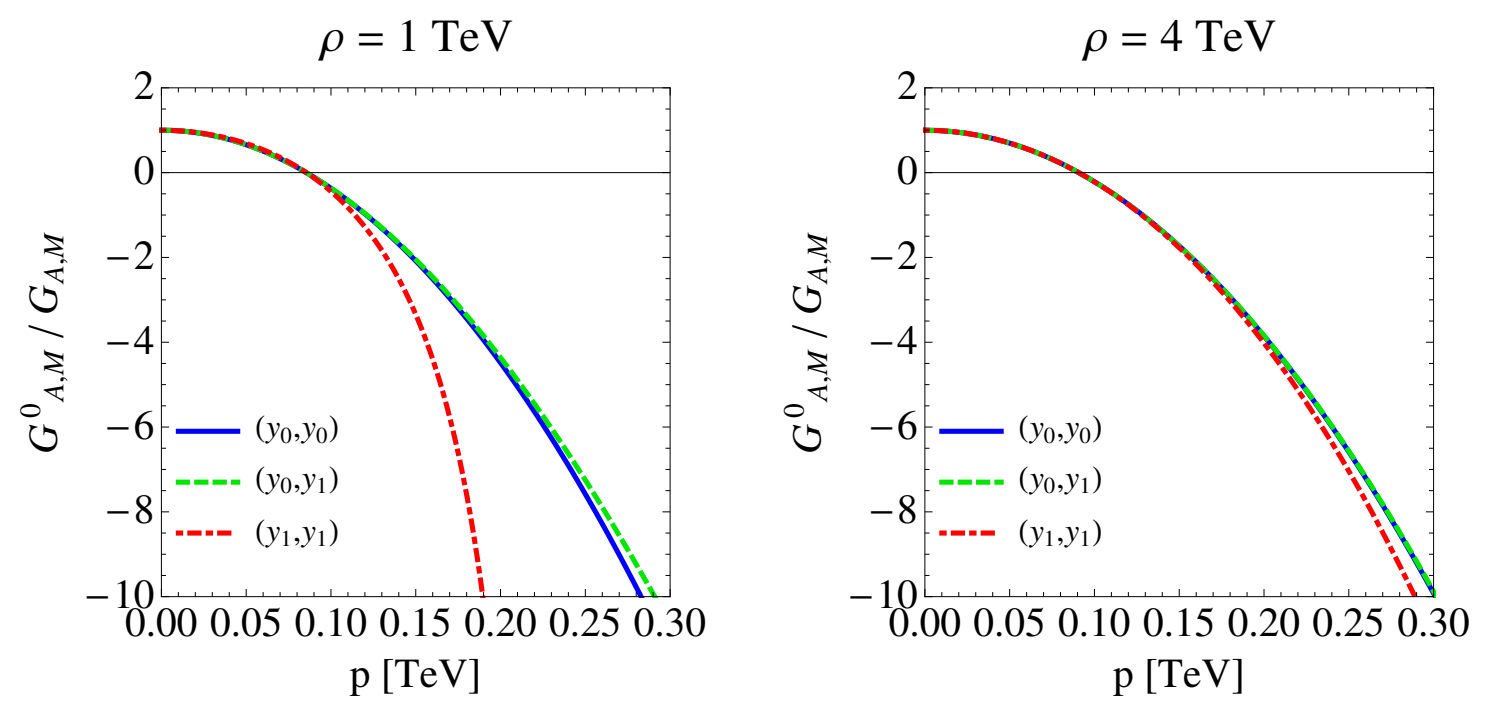

Figure 13. Inverse $\left(y_{0}, y_{0}\right),\left(y_{0}, y_{1}\right)$ and $\left(y_{1}, y_{1}\right)$ Green's functions for $A=Z, m_{Z}=0.091 \mathrm{TeV}$. They are normalized by their values in $p=0$. We display the results for $\rho=1 \mathrm{TeV}$ (left panel) and $\rho=4 \mathrm{TeV}$ (right panel). We have used $A_{1}=35$.

structure of zeros of this function, corresponding to resonances, can be seen. The lightest resonances appear at

$$
\begin{aligned}
(M / \rho, \Gamma / M)_{\rho=1 \mathrm{TeV}=} & (2.55,1.44),(5.91,0.670),(9.14,0.467),(12.34,0.366), \\
& (15.52,0.304), \cdots, \\
(M / \rho, \Gamma / M)_{\rho=4 \mathrm{TeV}=} & (2.35,2.86),(5.87,1.10),(9.14,0.744),(12.35,0.574), \\
& (15.54,0.472), \cdots .
\end{aligned}
$$

As it can be seen in figure 14, and from a comparison with eq. (3.43), the effect of the mass for the zero modes of the gauge bosons is to reduce the width of the resonances, but the masses of the resonances are not much affected. We display in figure 15 the dependence of the ratios $M / \rho$ (left panel) and $\Gamma / M$ (right panel) with the gauge boson mass for the three lightest resonances. Notice that for $\rho=1 \mathrm{TeV}$ the physical values for the $Z$ and $W$ masses correspond to $m_{A} / \rho \simeq 0.1$, and this leads to a reduction of the widths of the resonances by a factor $\sim 0.6$. A physical interpretation consistent with this property is that the KK modes are quasi bound states with a finite probability to tunnel to the continuum region $z>z_{1}$. As the brane mass of eq. (A.1) is taken larger, it tends to produce almost Dirichlet boundary conditions at the IR brane and the KK resonances become more stable, i.e. their widths tend to zero. ${ }^{17}$

We can study analytically the zeros of $\Phi_{M}(p)$ following the procedure of section 3.3. The expansion of the function $\Phi_{M}(p)$ at large momentum $\rho \ll|p|(|p| \ll k)$ and $1 \ll$

\footnotetext{
${ }^{17}$ We thank the (anonymous) referee for a useful comment on this issue.
} 

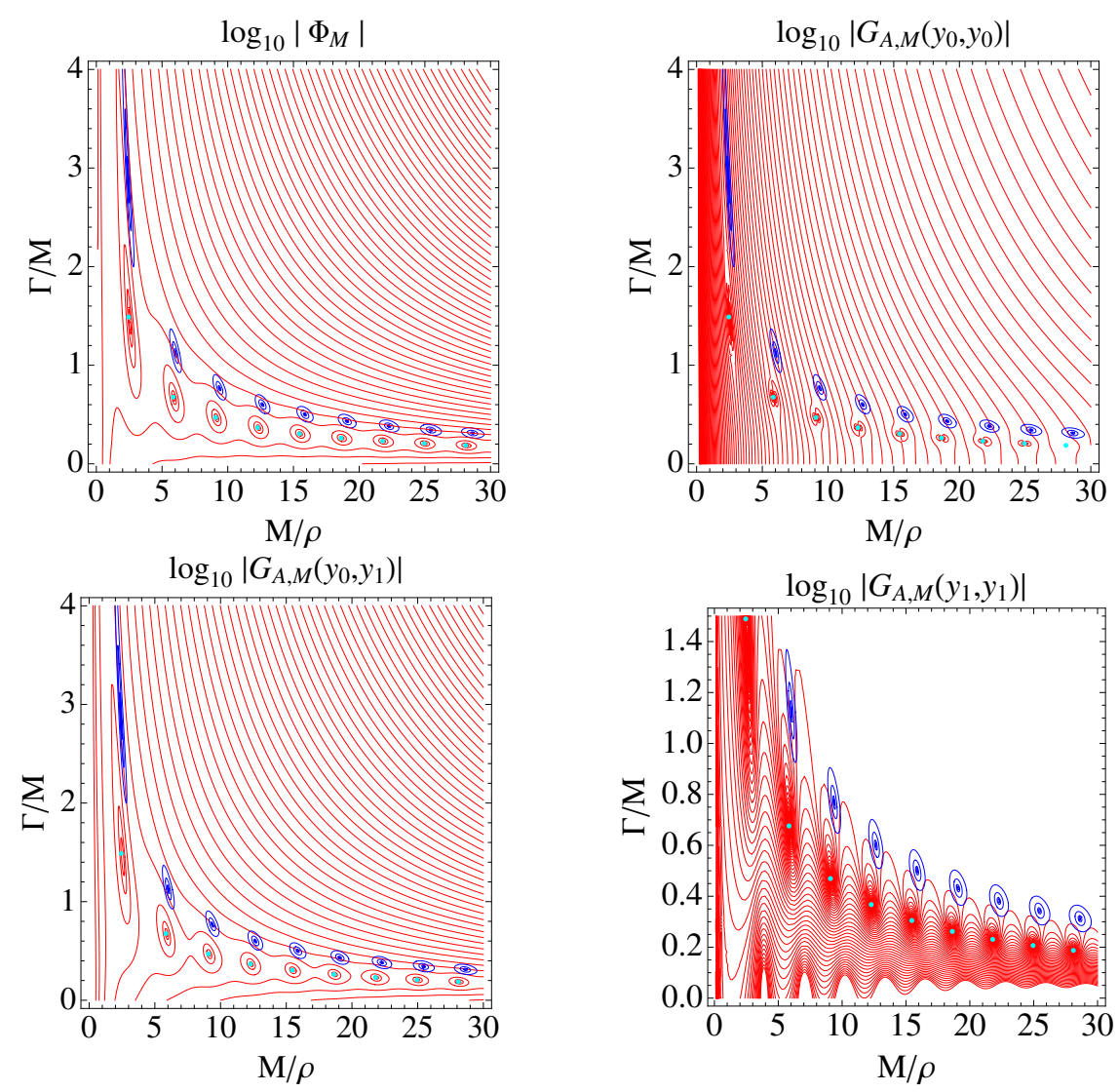

Figure 14. Upper left panel: contour plot in the plane $(M / \rho, \Gamma / M)$ of $\log _{10}\left|\Phi_{M}(p)\right|$ cf. eq. (A.4) (red lines). Upper right and lower panels: common logarithm of the absolute value of the Green's function $\log _{10}\left|G_{A, M}\left(y_{0}, y_{0}\right)\right|$ (upper right), $\log _{10}\left|G_{A, M}\left(y_{0}, y_{1}\right)\right|$ (lower left) and $\log _{10}\left|G_{A, M}\left(y_{1}, y_{1}\right)\right|$ (lower right). The (cyan) dots stand for the positions of the poles of the Green's function as predicted by the analytical formula of eq. (A.23). For comparison, the positions of the zeros of $\Phi(p)$ in the massless case, cf. figure 4 , are displayed in blue in each panel. We have considered $\rho=1 \mathrm{TeV}$, $m_{Z}=0.091 \mathrm{TeV}$ and $A_{1}=35$.

$k y_{s}\left(m_{A} / \rho\right)^{2} \cdot|p| / \rho \ll|p|^{2} / \rho^{2}$ leads to ${ }^{18}$

$$
\begin{gathered}
\Phi_{M}(p) \underset{\rho \ll|p|}{\simeq} 2 \sqrt{\frac{2}{\pi^{3}}} e^{-i(p / \rho-\pi / 4)}\left[k y_{s}\left(m_{A} / \rho\right)^{2} e^{i 2 p / \rho}-2 \frac{p}{\rho}\right] \log \left(\frac{p}{k}\right)\left(\frac{\rho}{p}\right)^{1 / 2}, \\
\operatorname{Im}\left((p / \rho)^{2}\right)<0 .
\end{gathered}
$$

${ }^{18}$ The large momentum expansion of the function $\Phi_{M}(p)$ is

$$
\Phi_{M}(p) \underset{\rho \ll|p|}{\propto} e^{i 2 p / \rho}\left(1+4 i k y_{s}\left(m_{A} / \rho\right)^{2} \cdot p / \rho\right)+4 p / \rho\left(-2 i p / \rho+k y_{s}\left(m_{A} / \rho\right)^{2}\right),
$$

for $\operatorname{Im}\left((p / \rho)^{2}\right)<0$, so that one can see that in the limit $m_{A} / \rho \rightarrow 0$ the massless case formula of eq. (3.44) is recovered. However, the zeros of this formula do not admit a direct analytical expression unless some of the terms are neglected. This is why in getting eq. (A.21) we have assumed $k y_{s}\left(m_{A} / \rho\right)^{2} \ll \frac{|p|}{\rho}$ and $1 \ll k y_{s}\left(m_{A} / \rho\right)^{2} \cdot \frac{|p|}{\rho}$, so that two of the terms in eq. (A.20) have been neglected. The disadvantage of this approximation is that the massless limit can no longer be recovered. 

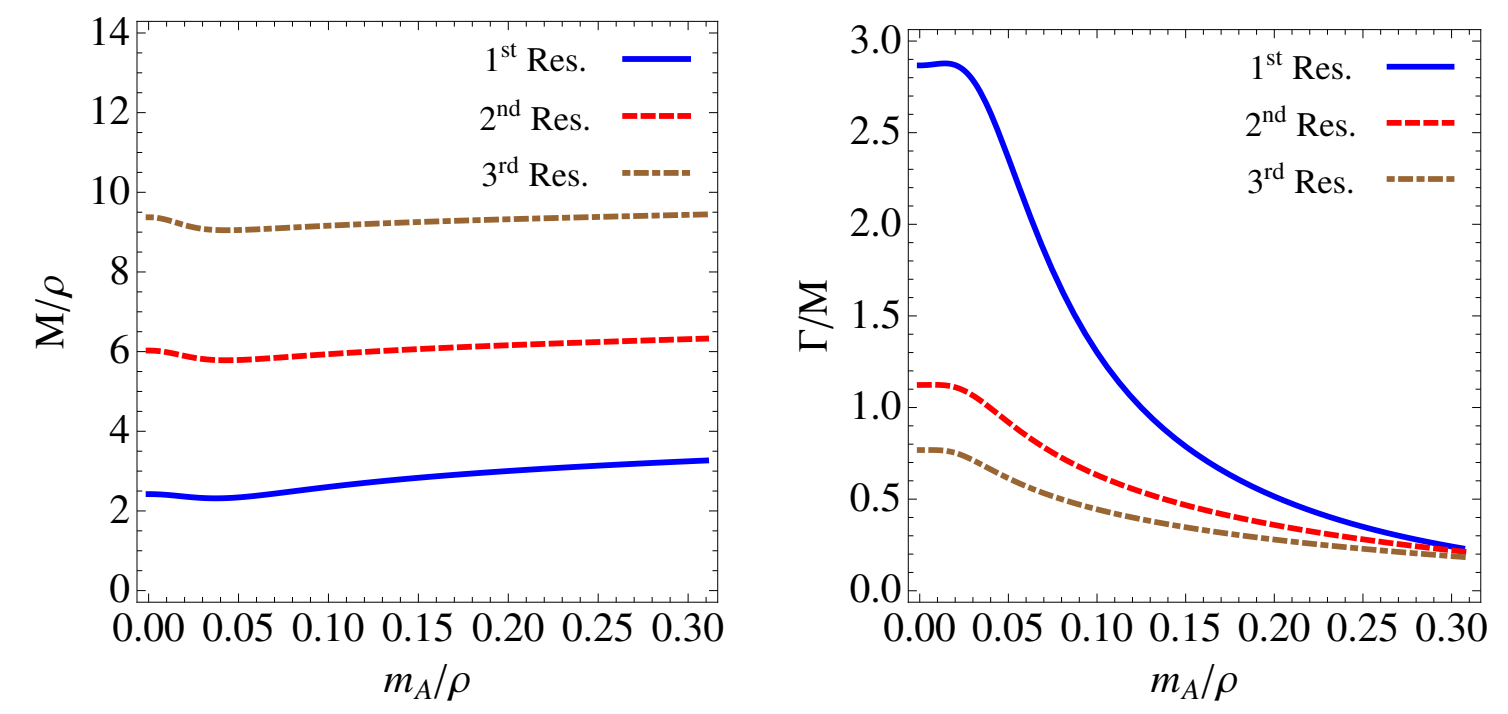

Figure 15. $M / \rho$ (left panel) and $\Gamma / M$ (right panel) as a function of the gauge boson mass $m_{A} / \rho$. It is displayed the results for the first (solid blue), second (dashed red) and third (dotdashed brown) resonances. We have considered $A_{1}=35$.

Then, the zeros of $\Phi_{M}(p)$ correspond to the solutions of the equation

$$
k y_{s}\left(m_{A} / \rho\right)^{2} e^{i 2 p / \rho}=2 \frac{p}{\rho},
$$

which turn out to be

$$
\left(\frac{p}{\rho}\right)^{2}=-\frac{1}{4} \mathcal{W}_{n}\left[-i k y_{s}\left(m_{A} / \rho\right)^{2}\right]^{2}, \quad n=-1,-2, \cdots
$$

We display in figure 14 as cyan dots the results of eq. (A.23) with $m_{A}=m_{Z}$ and $\rho=1 \mathrm{TeV}$. The relative error of eq. (A.23) with respect to the true zeros of $\Phi_{M}(p)$ decreases with $M / \rho$, and it is $\lesssim 0.5 \%$ except for the lightest resonance which is $\sim 4 \%$. The error of the approximate formula (A.23) increases when $m_{A} / \rho$ decreases as in this case the approximation $1 \ll k y_{s}\left(m_{A} / \rho\right)^{2} \cdot \frac{|p|}{\rho}$ is no longer valid. In this regime of very small gauge boson masses the formula of eq. (3.46) is a much better approximation.

Let us notice that the Green's function for massive gauge bosons can be split also into unparticle and resonant contributions, as for the massless case in eq. (3.65). The unparticle contribution for massive gauge bosons turns out to be identical as for massless gauge bosons, and it is given by eq. (3.66).

Finally, we show in figure 16 the brane-to-brane spectral functions $\rho_{A, M}\left(y_{\alpha}, y_{\beta} ; p\right)$ as functions of $p$, for $\rho=1 \mathrm{TeV}$ and $\rho=4 \mathrm{TeV}$. The prefactors, defined in eq. (3.31), make them approximately invariant under shifts of $k y_{s}$. In contrast to the results obtained in section 3.4 for massless gauge bosons, where it appears a Dirac delta behavior at $p=0$, in the massive case this behavior is found at $p \simeq m_{A}$. In all the cases the continuum spectrum starts at $p=0.5 \mathrm{TeV}(2 \mathrm{TeV})$ for $\rho=1 \mathrm{TeV}(4 \mathrm{TeV})$. 

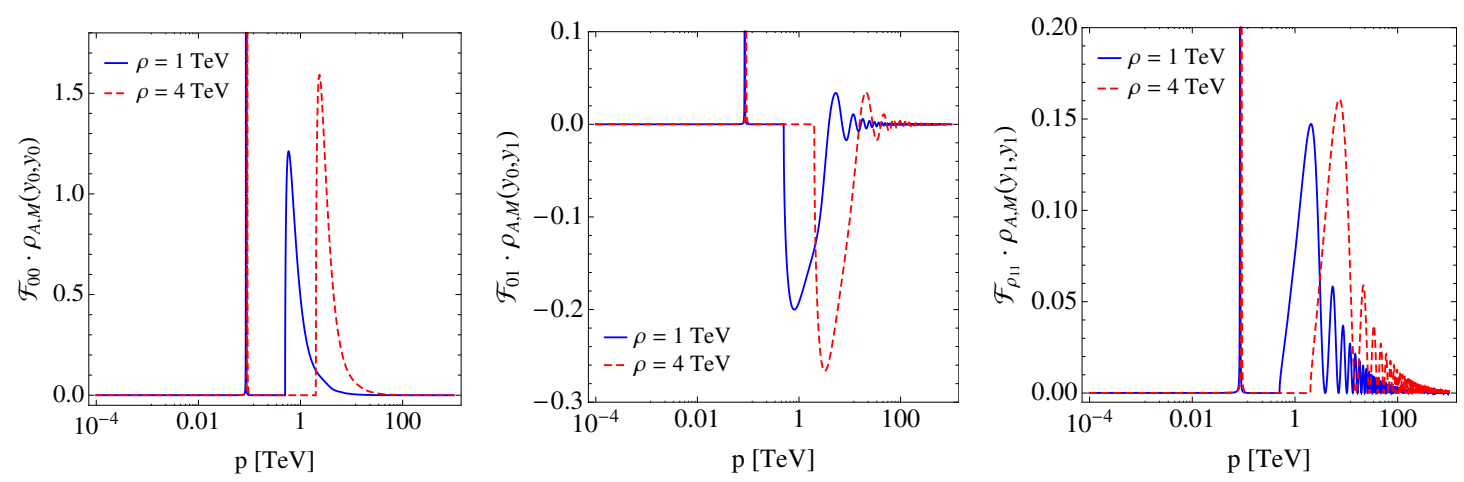

Figure 16. Rescaled spectral functions $\mathcal{F}_{00} \cdot \rho_{A, M}\left(y_{0}, y_{0} ; p\right)$ (left panel), $\mathcal{F}_{01} \cdot \rho_{A, M}\left(y_{0}, y_{1} ; p\right)$ (middle panel) and $\mathcal{F}_{11} \cdot \rho_{A, M}\left(y_{1}, y_{1} ; p\right)$ (right panel) for $A=Z, m_{Z}=0.091 \mathrm{TeV}$. We display the results for $\rho=1 \mathrm{TeV}$ (solid blue lines) and $\rho=4 \mathrm{TeV}$ (dashed red lines). We have used $A_{1}=35$ in all panels and assume time-like momenta $p^{2}>0$.

We can study as well the positivity of the spectral operator $\hat{\rho}_{A, M}$. The procedure is similar to the one presented in section 3.4 and the eigenvalue $\lambda_{M}(p)$ is given by

$$
\lambda_{M}(s)=\delta\left(s-m_{A}^{2}\right)+\left[-\frac{\log \bar{\epsilon}}{2 \pi \rho} \lambda_{\text {un }}(s)+\mathcal{O}\left(\bar{\epsilon}^{0}\right)\right] \Theta\left(s-m_{g}^{2}\right), \quad \lambda_{\text {un }}(s)=\left(s-m_{g}^{2}\right)^{-1 / 2},
$$

reflecting the existence of the isolated zero mode with squared mass $m_{A}^{2} \ll m_{g}^{2}$.

Open Access. This article is distributed under the terms of the Creative Commons Attribution License (CC-BY 4.0), which permits any use, distribution and reproduction in any medium, provided the original author(s) and source are credited.

\section{References}

[1] ALEPH, DELPHI, L3, OPAL, SLD, LEP Electroweak Working Group, SLD Electroweak Group and SLD Heavy Flavour Group collaborations, Precision electroweak measurements on the $Z$ resonance, Phys. Rept. 427 (2006) 257 [hep-ex/0509008] [INSPIRE].

[2] Particle Data Group collaboration, Review of Particle Physics, Chin. Phys. C 40 (2016) 100001 [INSPIRE].

[3] L. Randall and R. Sundrum, A large mass hierarchy from a small extra dimension, Phys. Rev. Lett. 83 (1999) 3370 [hep-ph/9905221] [INSPIRE].

[4] CMS collaboration, Search for resonant $\mathrm{t} \overline{\mathrm{t}}$ production in proton-proton collisions at $\sqrt{s}=13 \mathrm{TeV}$, JHEP 04 (2019) 031 [arXiv: 1810.05905] [INSPIRE].

[5] ATLAS collaboration, Search for heavy particles decaying into a top-quark pair in the fully hadronic final state in pp collisions at $\sqrt{s}=13 \mathrm{TeV}$ with the ATLAS detector, Phys. Rev. D 99 (2019) 092004 [arXiv: 1902.10077] [INSPIRE].

[6] R. Escribano, M. Mendizabal, M. Quirós and E. Royo, On Broad Kaluza-Klein Gluons, JHEP 05 (2021) 121 [arXiv:2102.11241] [INSPIRE]. 
[7] G.F. Giudice and M. McCullough, Comment on "Disassembling the Clockwork Mechanism", arXiv:1705.10162 [INSPIRE].

[8] G.F. Giudice, Y. Kats, M. McCullough, R. Torre and A. Urbano, Clockwork/linear dilaton: structure and phenomenology, JHEP 06 (2018) 009 [arXiv:1711.08437] [INSPIRE].

[9] I. Antoniadis, A. Arvanitaki, S. Dimopoulos and A. Giveon, Phenomenology of TeV Little String Theory from Holography, Phys. Rev. Lett. 108 (2012) 081602 [arXiv:1102.4043] [INSPIRE].

[10] P. Cox and T. Gherghetta, Radion Dynamics and Phenomenology in the Linear Dilaton Model, JHEP 05 (2012) 149 [arXiv:1203.5870] [InSPIRE].

[11] I. Antoniadis, S. Dimopoulos and A. Giveon, Little string theory at a TeV, JHEP 05 (2001) 055 [hep-th/0103033] [INSPIRE].

[12] C. Csáki, G. Lee, S.J. Lee, S. Lombardo and O. Telem, Continuum Naturalness, JHEP 03 (2019) 142 [arXiv: 1811.06019] [INSPIRE].

[13] E. Megías and M. Quirós, Gapped Continuum Kaluza-Klein spectrum, JHEP 08 (2019) 166 [arXiv: 1905. 07364] [INSPIRE].

[14] E. Megías and M. Quirós, On gapped continuum resonance spectra, in 8th International Conference on New Frontiers in Physics, (2020) [arXiv:2002.11756] [INSPIRE].

[15] E. Megías and M. Quirós, The Continuum Linear Dilaton, Acta Phys. Polon. B 52 (2021) 711 [arXiv:2104.10260] [INSPIRE].

[16] C. Csáki, S. Hong, G. Kurup, S.J. Lee, M. Perelstein and W. Xue, Continuum Dark Matter, arXiv:2105.07035 [INSPIRE].

[17] J.A. Cabrer, G. von Gersdorff and M. Quirós, Warped Electroweak Breaking Without Custodial Symmetry, Phys. Lett. B 697 (2011) 208 [arXiv:1011.2205] [INSPIRE].

[18] J.A. Cabrer, G. von Gersdorff and M. Quirós, Suppressing Electroweak Precision Observables in 5D Warped Models, JHEP 05 (2011) 083 [arXiv:1103.1388] [INSPIRE].

[19] J.A. Cabrer, G. von Gersdorff and M. Quirós, Improving Naturalness in Warped Models with a Heavy Bulk Higgs Boson, Phys. Rev. D 84 (2011) 035024 [arXiv:1104.3149] [InSPIRE].

[20] J.A. Cabrer, G. von Gersdorff and M. Quirós, Warped 5D Standard Model Consistent with EWPT, Fortsch. Phys. 59 (2011) 1135 [arXiv:1104.5253] [INSPIRE].

[21] J.A. Cabrer, G. von Gersdorff and M. Quirós, Flavor Phenomenology in General 5D Warped Spaces, JHEP 01 (2012) 033 [arXiv:1110.3324] [INSPIRE].

[22] J.A. Cabrer, G. von Gersdorff and M. Quirós, Soft-Wall Stabilization, New J. Phys. 12 (2010) 075012 [arXiv:0907.5361] [inSPIRE].

[23] H. Georgi, Unparticle physics, Phys. Rev. Lett. 98 (2007) 221601 [hep-ph/0703260] [INSPIRE].

[24] H. Georgi, Another odd thing about unparticle physics, Phys. Lett. B 6550 (2007) 275 [arXiv: 0704.2457] [INSPIRE].

[25] A. Delgado, J.R. Espinosa and M. Quirós, Unparticles Higgs Interplay, JHEP 10 (2007) 094 [arXiv: 0707.4309] [INSPIRE].

[26] A. Delgado, J.R. Espinosa, J.M. No and M. Quirós, The Higgs as a Portal to Plasmon-like Unparticle Excitations, JHEP 04 (2008) 028 [arXiv:0802.2680] [INSPIRE]. 
[27] A. Delgado, J.R. Espinosa, J.M. No and M. Quirós, A Note on Unparticle Decays, Phys. Rev. D 79 (2009) 055011 [arXiv: 0812.1170] [INSPIRE].

[28] A. Delgado, J.R. Espinosa, J.M. No and M. Quirós, Phantom Higgs from Unparticles, JHEP 11 (2008) 071 [arXiv:0804.4574] [INSPIRE].

[29] D. Stancato and J. Terning, The Unhiggs, JHEP 11 (2009) 101 [arXiv:0807.3961] [INSPIRE].

[30] A. Falkowski and M. Pérez-Victoria, Holographic Unhiggs, Phys. Rev. D 79 (2009) 035005 [arXiv:0810.4940] [INSPIRE].

[31] A. Falkowski and M. Pérez-Victoria, Electroweak Precision Observables and the Unhiggs, JHEP 12 (2009) 061 [arXiv:0901.3777] [INSPIRE].

[32] B. Bellazzini, C. Csáki, J. Hubisz, S.J. Lee, J. Serra and J. Terning, Quantum Critical Higgs, Phys. Rev. X 6 (2016) 041050 [arXiv:1511.08218] [inSPIRE].

[33] K. Agashe, A. Delgado, M.J. May and R. Sundrum, RS1, custodial isospin and precision tests, JHEP 08 (2003) 050 [hep-ph/0308036] [INSPIRE].

[34] S.S. Gubser, AdS/CFT and gravity, Phys. Rev. D 63 (2001) 084017 [hep-th/9912001] [INSPIRE].

[35] J.W. York Jr., Role of conformal three geometry in the dynamics of gravitation, Phys. Rev. Lett. 28 (1972) 1082 [INSPIRE].

[36] G.W. Gibbons and S.W. Hawking, Action Integrals and Partition Functions in Quantum Gravity, Phys. Rev. D 15 (1977) 2752 [InSPIRE].

[37] E. Megías, G. Nardini and M. Quirós, Cosmological Phase Transitions in Warped Space: Gravitational Waves and Collider Signatures, JHEP 09 (2018) 095 [arXiv: 1806. 04877] [INSPIRE].

[38] O. DeWolfe, D.Z. Freedman, S.S. Gubser and A. Karch, Modeling the fifth-dimension with scalars and gravity, Phys. Rev. D 62 (2000) 046008 [hep-th/9909134] [InSPIRE].

[39] C. Csáki, M.L. Graesser and G.D. Kribs, Radion dynamics and electroweak physics, Phys. Rev. D 63 (2001) 065002 [hep-th/0008151] [INSPIRE].

[40] C. Csáki, TASI lectures on extra dimensions and branes, in Theoretical Advanced Study Institute in Elementary Particle Physics (TASI 2002): Particle Physics and Cosmology: The Quest for Physics Beyond the Standard Model(s), (2004), pp. 605-698 [hep-ph/0404096] [INSPIRE].

[41] R.N. Mohapatra and J.C. Pati, Left-Right Gauge Symmetry and an Isoconjugate Model of CP-violation, Phys. Rev. D 11 (1975) 566 [inSPIRE].

[42] R.N. Mohapatra and J.C. Pati, A Natural Left-Right Symmetry, Phys. Rev. D 11 (1975) 2558 [INSPIRE].

[43] G. Senjanović and R.N. Mohapatra, Exact Left-Right Symmetry and Spontaneous Violation of Parity, Phys. Rev. D 12 (1975) 1502 [InSPIRE].

[44] M. Carena, E. Megías, M. Quirós and C. Wagner, $R_{D^{(*)}}$ in custodial warped space, JHEP 12 (2018) 043 [arXiv : 1809.01107] [INSPIRE].

[45] T. Wolkanowski, Resonances and poles in the second Riemann sheet, arXiv:1303.4657 [INSPIRE]. 
[46] D.T. Son and A.O. Starinets, Minkowski space correlators in AdS/CFT correspondence: Recipe and applications, JHEP 09 (2002) 042 [hep-th/0205051] [INSPIRE].

[47] A. Costantino and S. Fichet, Opacity from Loops in AdS, JHEP 02 (2021) 089 [arXiv: 2011.06603] [INSPIRE].

[48] P.V. Landshoff, Poles and Thresholds and Unstable Particles, Nuovo Cim. 28 (1963) 123.

[49] E. Megías, M. Pérez-Victoria and M. Quirós, work in progress (2021).

[50] M.E. Peskin and T. Takeuchi, Estimation of oblique electroweak corrections, Phys. Rev. D 46 (1992) 381 [INSPIRE].

[51] Particle Data Group collaboration, Review of Particle Physics, PTEP 2020 (2020) 083C01 [INSPIRE].

[52] I. Chaffey, S. Fichet and P. Tanedo, Continuum-Mediated Self-Interacting Dark Matter, JHEP 06 (2021) 008 [arXiv:2102.05674] [INSPIRE]. 